\title{
Flexible dynamics of two quorum-sensing coupled repressilators
}

\author{
Edward H. Hellen* \\ Department of Physics \& Astronomy, University of North Carolina Greensboro, Greensboro, North Carolina, USA
}

Evgeny Volkov

Department of Theoretical Physics, Lebedev Physical Institute, Moscow, Russia

(Received 24 August 2016; revised manuscript received 23 January 2017; published 17 February 2017)

\begin{abstract}
Genetic oscillators play important roles in cell life regulation. The regulatory efficiency usually depends strongly on the emergence of stable collective dynamic modes, which requires designing the interactions between genetic networks. We investigate the dynamics of two identical synthetic genetic repressilators coupled by an additional plasmid which implements quorum sensing (QS) in each network thereby supporting global coupling. In a basic genetic ring oscillator network in which three genes inhibit each other in unidirectional manner, QS stimulates the transcriptional activity of chosen genes providing for competition between inhibitory and stimulatory activities localized in those genes. The "promoter strength", the Hill cooperativity coefficient of transcription repression, and the coupling strength, i.e., parameters controlling the basic rates of genetic reactions, were chosen for extensive bifurcation analysis. The results are presented as a map of dynamic regimes. We found that the remarkable multistability of the antiphase limit cycle and stable homogeneous and inhomogeneous steady states exists over broad ranges of control parameters. We studied the antiphase limit cycle stability and the evolution of irregular oscillatory regimes in the parameter areas where the antiphase cycle loses stability. In these regions we observed developing complex oscillations, collective chaos, and multistability between regular limit cycles and complex oscillations over uncommonly large intervals of coupling strength. QS coupling stimulates the appearance of intrachaotic periodic windows with spatially symmetric and asymmetric partial limit cycles which, in turn, change the type of chaos from a simple antiphase character into chaos composed of pieces of the trajectories having alternating polarity. The very rich dynamics discovered in the system of two identical simple ring oscillators may serve as a possible background for biological phenotypic diversification, as well as a stimulator to search for similar coupling in oscillator arrays in other areas of nature, e.g., in neurobiology, ecology, climatology, etc.
\end{abstract}

DOI: 10.1103/PhysRevE.95.022408

\section{INTRODUCTION}

Synthetic genetic networks provide researchers with the opportunity of designing biologically based circuitry for accomplishing specific functions. Additionally, networks displaying multistable dynamics provide a method for investigating possible mechanisms for biological diversity. In principle, such circuits can be incorporated into natural cellular machinery or used in an entirely synthetic environment. Oscillators are essential motifs for circuit design [1]. The repressilator is a synthetic genetic oscillator (GO) in the form of a ring of three genes sequentially inhibiting one another's transcription. The GO has been inserted experimentally into E. coli [2] and extensively studied theoretically via deterministic and stochastic approaches [3-5].

Coupling of individual GOs is important in coordinated activity of GO populations. Bacterial quorum sensing (QS) [6], which provides for cell-cell communications in bacterial populations by fast diffusion of small specific molecules (autoinducers), is a natural candidate for the role of being

\footnotetext{
*ehhellen@uncg.edu
}

Published by the American Physical Society under the terms of the Creative Commons Attribution 3.0 License. Further distribution of this work must maintain attribution to the author(s) and the published article's title, journal citation, and DOI. a manager for synthetic genetic network dynamics. This idea was effective, for instance, in constructing several synthetic multicellular systems like the ecological predator-prey [7], population control [8], and other models (see references in a recent review [9]).

QS coupling has been used for GO synchronization in different mathematical models [10-12], as well as in experimental demonstration of a multicellular clock [13]. Recently the QS system has been used to construct a synthetic microbial consortium with population-level oscillations [14]. It has been shown that the specific properties of isolated synthetic oscillators, e.g., the choice between "soft" and "stiff" limit cycles, and the type of coupling, repulsive or attractive, have great influence on the resulting collective modes [15]. Great variability of collective dynamics has already been observed, but the mechanisms of their emergence have been only partially revealed. One opinion is that the spectrum of dynamical regimes is determined by the fixed type of single element used in designing a genetic network. However, this opinion is not always correct, even for isolated genetic clocks in which "rate constants rather than biochemical mechanism determine behavior" [16]. Therefore, the role of particular parameters in the given QS-linked genetic network requires special attention, as we do here. Although autoinducers freely diffuse between cells with GOs inside, this type of coupling as a whole cannot be reduced to simple linear diffusive coupling, which still dominates in studies of coupled oscillators. An autoinducer is not a required element of a $\mathrm{GO}$; its production may be controlled by one gene of the GO 
networks, but its target is transcription regulation of a different gene in the same or neighboring cells. Being physically diffusive, this coupling mechanism is, however, difficult to present as just one classical diffusive term in the ODE system describing the genetic circuits. To write the coupling term mathematically it is necessary to consistently consider the particular reaction scheme. This way to draw the coupling term seems quite natural for biochemical and genetic networks in which communication is a cumulative multistep process which includes regulative production, diffusion, binding to targets, and other important metabolic steps.

The first attempt to demonstrate theoretically the possibility of in-phase synchronization of repressilators was successfully realized [11] and checked in a simple electronic model [17], but not all collective regimes were detected within the limits of the coupling design considered. Later publication demonstrated that the use of limited parameter space masked other dynamical regimes. The antiphase limit cycle and complex periodic regimes were revealed when stronger repression in transcriptional regulation and greater differences in time scales of mRNA and transcription factor kinetics were taken into account [18].

In the next version [19,20] of QS-dependent cell-cell interaction, identical repressilators were coupled using a modification of the additional plasmid for the QS mechanism. The modification provided phase-repulsive interaction between oscillators, which leads to a rich set of stable attractors: a periodic regular antiphase limit cycle (APLC: time series are shifted by half-period), a stable homogeneous steady state (HSS: the identical values of the same name variables in each oscillator), inhomogeneous steady states (IHSS: different values of variables), an inhomogeneous limit cycle (IHLC) emerging from IHSS, and a chaotic regime which appears via torus bifurcation of the APLC branch. Some regimes can coexist over broad intervals of model parameters, which opens a possibility for switching between attractors in the presence of extrinsic or intrinsic noise. Specific examples of all these regimes will be presented in the Results section.

An important problem in the studies of gene expression regulation is very limited knowledge of the parameter values. The transcription regulation is typically described by the Hill function, $\alpha /\left(1+x^{n}\right)$, where the main parameters are the maximum transcription rate $(\alpha)$ and the degree of cooperativity $(n)$ of the transcription factor $(x)$ binding to promoter. Previous publications $[19,20]$ concentrated on the dynamics with small Hill coefficient $n$ and limited values for transcription rates, time scales ratios for mRNA and proteins kinetics, and QS signaling molecule (autoinducer) activity as transcription activator. The goals of this paper are to significantly extend the main parameter areas within the limits of one model of QS-coupled identical or nearly identical repressilators [20] to detect new dynamic behavior(s), to present the coarse-grained structure and content of the phase diagram (the map of regimes), and to investigate the robustness of multistability with respect to parameter values. We will use a reduced version of the mathematical model, as well as its electronic circuit model [21,22] adapted for two coupled 4-dim repressilators. The effectiveness of this analog version has been checked recently in the study of a single repressilator with QS feedback [23]. Use of the inherently different numerical and electronic models provides a test of the robustness of the dynamics. We consider a wide range of control parameters, even going beyond the characteristic limits known for them today. The results obtained using the extended ranges may be viewed as predictions of the model for the future, when these extended limits become accessible with the fast development of synthetic genetics.

The dynamical results produced by the QS-coupled oscillators used here may be predictive for populations of cells in the context of repulsive coupling, which has been shown to be capable of splitting a population into a small number of clusters [24]. These results may also inspire application to other systems beyond synthetic gene circuits, such as electronic ones (see below) or chemical systems of interacting water droplets bearing oscillating $\mathrm{BZ}$ reactions coupled via diffusion in selective oil environments [25].

We find that for a small degree of repression of transcription factor binding (Hill coefficient $n<3$ ) APLC is the single homogeneous periodic attractor, and that this attractor loses stability via torus bifurcation if $n$ increases $(n=3)$ and gets replaced by complex or chaotic regimes over a large interval of coupling strengths $(Q)$. This $Q$-continuation branch of APLC and complex oscillations coexists with HSS, IHSS, and IHLC, providing a rich landscape of attractors in multiparameter space.

The sizes of a parameter's intervals occupied by the complex regimes are unusually large and include many periodic windows which contain both spatially symmetrical and asymmetrical stable limit cycles. The presence of asymmetrical limit cycles results in the appearance of chaotic trajectories built from pieces with randomly alternating "polarity". This type of chaotic time series differs from that of simple antiphase chaos observed in parameter regions devoid of asymmetrical limit cycles.

The extension of the ranges for parameter analysis also reveals another type of multistability if changes in the maximum transcription rate $(\alpha)$ are taken into account. For a value of the Hill coefficient near three a range of $\alpha$ gives rise to the coexistence of the complex nonperiodic oscillations and an APLC with five return times in one period. Again, the range of this hysteresis covers large intervals of the coupling strengths and $\alpha$.

A further increase in transcription cooperativity up to $n=4$ leads to the recovery of stability of the in-phase limit cycle in addition to stable APLC. An in-phase limit cycle is not a dominant regime, but its appearance seems important to complete the entire dynamic picture demonstrated by the two coupled identical repressilators.

\section{NUMERICAL AND ELECTRICAL MODELS}

We investigate the dynamics of two repressilators interacting via repressive QS coupling as used previously [19,20]. Figure 1 shows a single repressilator coupled via QS to the external medium. The three genes in the loop produce mRNA $(a, b, c)$ and proteins $(A, B, C)$, and they impose Hill function inhibition on each other in cyclic order by the preceding gene. The QS feedback is maintained by the autoinducer (AI) produced (rate $k_{S 1}$ ) by the protein $\mathrm{B}$ while the AI communicates with the external environment and activates 


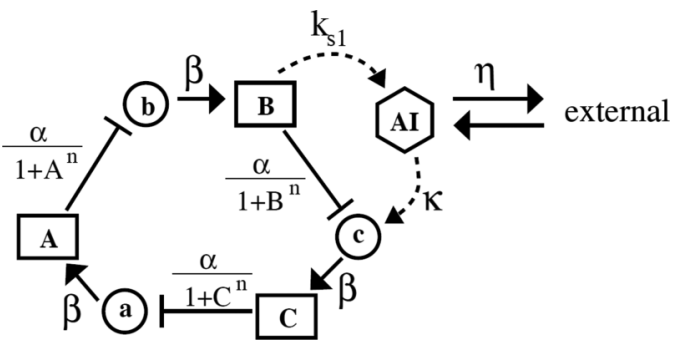

FIG. 1. A repressilator genetic network with QS feedback. Lower case $(\mathrm{a}, \mathrm{b}, \mathrm{c})$ are mRNA, and upper case $(\mathrm{A}, \mathrm{B}, \mathrm{C})$ are expressed protein repressors. AI is the autoinducer molecule which diffuses through the cell membrane.

(rate $\kappa$ in combination with Michaelis function) production of mRNA for protein $\mathrm{C}$, which, in turn, reduces the concentration of protein A resulting in activation of protein $\mathrm{B}$ production. In this way the protein B plays a dual role of direct inhibition of protein $\mathrm{C}$ synthesis and $\mathrm{AI}-$ dependent activation of protein $\mathrm{C}$ synthesis, resulting in complex dynamics of the repressilator, even for just a single repressilator [23].

The original models of a single repressilator [2,19] used rescaled dimensionless quantities for rate constants and concentrations. We reduce the model for the case of fast mRNA kinetics $[(a, b, c)$ assumed in steady state with their respective inhibitors $(C, A, B)$, so that $d a / d t=d b / d t=d c / d t \approx 0]$. The resulting equations for the protein concentrations and $\mathrm{AI}$ concentration $S$ are

$$
\begin{aligned}
\frac{d A_{i}}{d t} & =\beta_{1}\left(-A_{i}+\frac{\alpha}{1+C_{i}^{n}}\right), \\
\frac{d B_{i}}{d t} & =\beta_{2}\left(-B_{i}+\frac{\alpha}{1+A_{i}^{n}}\right), \\
\frac{d C_{i}}{d t} & =\beta_{3}\left(-C_{i}+\frac{\alpha}{1+B_{i}^{n}}+\frac{\kappa S_{i}}{1+S_{i}}\right), \\
\frac{d S_{i}}{d t} & =-k_{S 0} S_{i}+k_{S 1} B_{i}-\eta\left(S_{i}-S_{\mathrm{ext}}\right),
\end{aligned}
$$

where $i=1,2$ for the two repressilators, $\beta_{j}(j=1,2,3)$ are the ratios of the protein decay rate to mRNA decay rate, $\alpha$ accounts for the maximum transcription rate in the absence of an inhibitor, and $n$ is the Hill cooperativity coefficient for inhibition. For the quorum sensing pathway $k_{S 0}$ is the ratio of the AI decay rate to the mRNA decay rate, and as previously mentioned, $k s_{1}$ is the rate of production of AI and $\kappa$ gives the strength of AI activation of protein $\mathrm{C}$. The diffusion coefficient $\eta$ depends on the permeability of the membrane to the AI molecule. The concentration of AI in the external medium is $S_{\text {ext }}$ and is determined according to quasi-steady-state approximation by AI produced by both repressilators $\left(S_{1}\right.$ and $\left.S_{2}\right)$ and a dilution factor $Q$ :

$$
S_{\mathrm{ext}}=Q \frac{S_{1}+S_{2}}{2} .
$$

Numerical simulations are performed with XPPAUT [26] and by direct integration with a fourth-order Runge-Kutta solver. We choose parameter values similar to ones used previously shown to be experimentally reasonable taking into account realistic biochemical rates and binding affinities [20]. Here we use $\beta_{1}=0.5, \beta_{2}=\beta_{3}=0.1, n=2.8$ to $4, \alpha \approx 200, k_{S 0}=$ $1, k_{S 1}=0.01$, and $\eta=2$.

We also implement the model (1) in electronic circuits as described previously [21,23], with additional performance improvements [22]. By its very nature the circuit has inherent parameter mismatch and noise and is free from any numerical artifacts. It provides experimental results which complement numerical simulations. Model parameter values are set in the circuit by resister values, capacitor values, and reference voltages as described in the Appendix A. The hyperbolic dependence $S /(1+S)$ in Eq. (1c) is replaced in the circuit by the linear-piecewise-continuous behavior $\min (0.8 S, 1)$ as described previously [23]. Therefore Eq. (1c) in simulations is replaced by

$$
\frac{d C_{i}}{d t}=\beta_{3}\left[-C_{i}+\frac{\alpha}{1+B_{i}^{n}}+\kappa \min \left(0.8 S_{i}, 1\right)\right] .
$$

An important goal of the circuit design is to reproduce from Eqs. (1) and (3) the Hill function inhibition $1 /\left(1+x^{n}\right)$ and the QS activation $\min (0.8 S, 1)$. Figure 2 shows the resulting comparison of measured circuit performance and mathematical model for inhibition and activation.

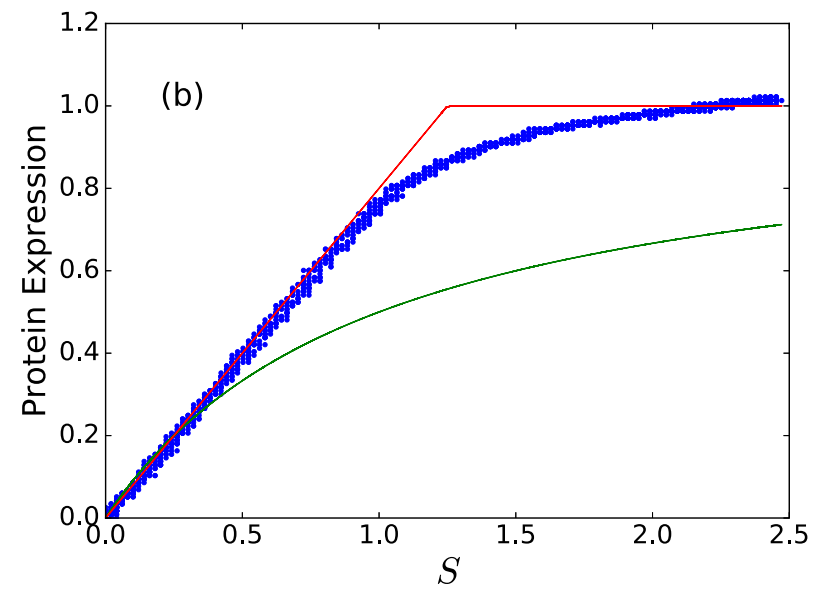

FIG. 2. Comparison of circuit behavior (blue dots) and mathematical model (red line) for (a) Hill inhibition for $n=3.17, \alpha=218$, and (b) QS activation for $\kappa=21, \alpha=135$. Panel (b) also shows $S /(1+S)$ as a green line. 

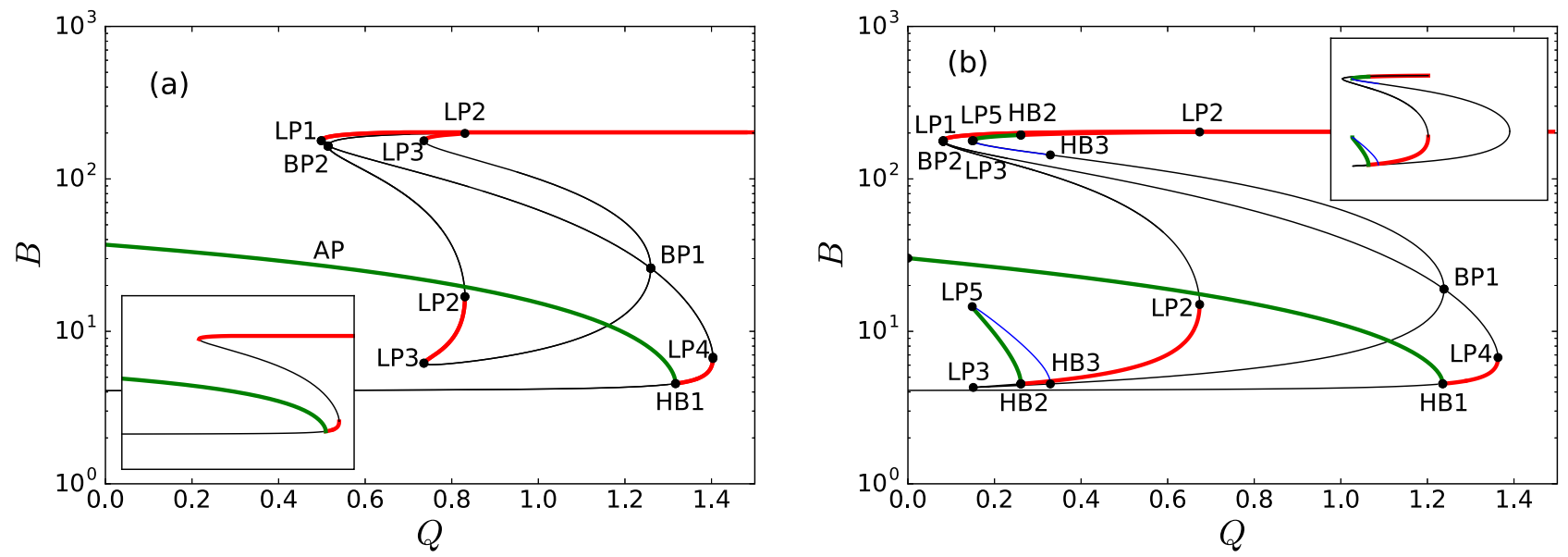

FIG. 3. Effect of increasing the QS activation $\kappa$. Numerical $Q$ continuations for two QS-coupled repressilators $(n=2.8, \alpha=204)$ showing the values of proteins $B_{i}$ for steady states and the amplitudes of oscillations for limit cycles. HB, Andronov-Hopf bifurcation; LP, limit point; BP, branch point (symmetry-breaking bifurcation). Stable (thick red) and unstable (thin black) steady state, stable (thick green) and unstable (thin blue) limit cycle. Two red lines between LP3 and LP2 correspond to values $B_{1}$ and $B_{2}$ for IHSS. (a) $\kappa=12$. Inset shows just the homogeneous branch. (b) $\kappa=17$. Inset shows just the inhomogeneous branch. (b) Two green lines between LP5 and HB2 are amplitudes $B_{1}$ and $B_{2}$ for stable IHLC, and two blue lines between LP5 and HB3 are for unstable IHLC.

\section{RESULTS}

\section{A. Low repression: $n=2.8$}

We start with the demonstration of the general structure of the phase diagram (map of regimes) for two coupled 4-dim repressilators [Eq. (1)] with parameters similar to those used previously [20] in a study with nonreduced coupled 7-dim repressilators. We choose protein $\mathrm{B}$ as the dynamical variable of interest.

Figure 3 shows bifurcation diagrams with Hill coefficient $n=2.8$ for two different activation rates, $\kappa=12$ and 17 . In these figures the coupling strength $Q$ is varied and the protein B maxima of resulting dynamical regimes are plotted: stable steady state (thick red), unstable steady state (thin black), stable LC (thick green), and unstable LC (thin blue). There are homogeneous dynamics in which both repressilators have the same maximum value and inhomogeneous (IH) dynamics

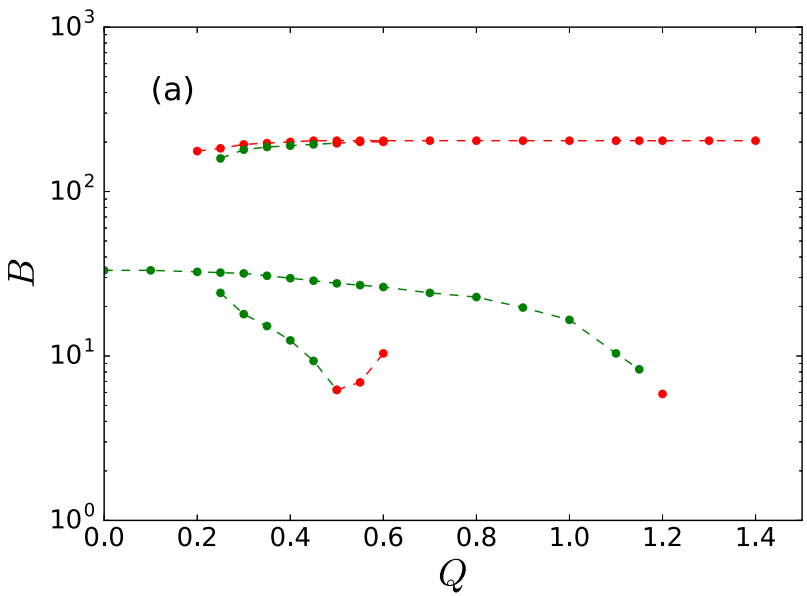

where they have different maximum values. For clarity, the homogeneous branch is shown alone in the inset in Fig. 3(a) and the IH branch is shown alone in the inset in Fig. 3(b). The branch points (BP1 and BP2 in Fig. 3) are where the homogeneous and IH steady-state branches intersect. The coupling strength is varied from 0 to 1.5 in order to show the entire structure of the system, although values of $Q>1$ are not accessible in the conventional biological system.

Figure 3(a) for activation strength $\kappa=12$ shows a variety of dynamical behaviors. The main features are the stabilization of the high-B-HSS for large coupling strength $(Q>0.5)$; APLC which is the only stable dynamical behavior for small coupling strength ( $Q$ below LP1) and which remains stable out to large coupling strength at HB1 where the APLC converts to a low-B-HSS; and the broken symmetry bifurcations (BP1 and BP2) where IH solutions arise including the stable IHSS between LP2 and LP3. There is coexistence of HSS, IHSS,

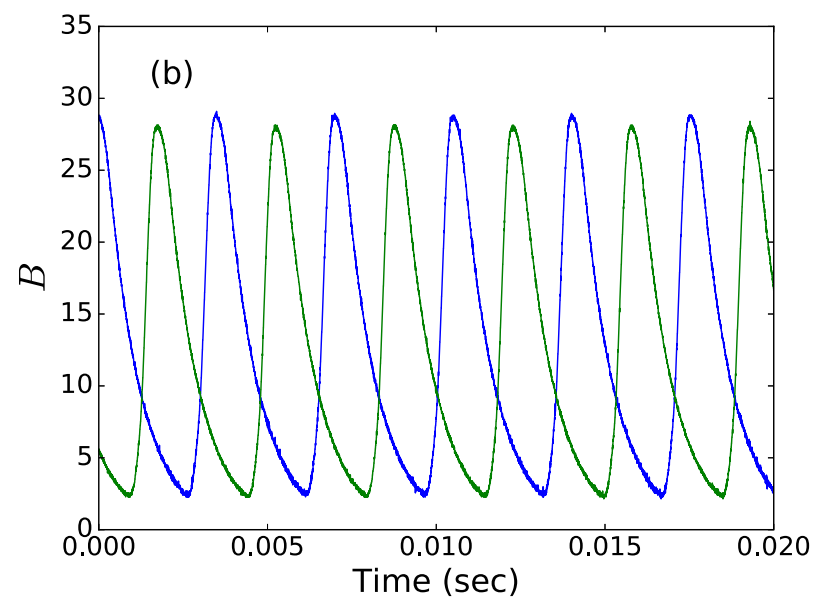

FIG. 4. Circuit measurements for two QS-coupled repressilators ( $n=2.8, \alpha=204, \kappa=17.8)$. (a) $Q$ continuation showing stable steadystates (red) and stable limit cycles (green). (b) Time series showing APLC for $Q=0.4$. $B$ values were calculated from measured voltages. Note that the APLC is continuously stable from $Q=0$ to 1.15 . 

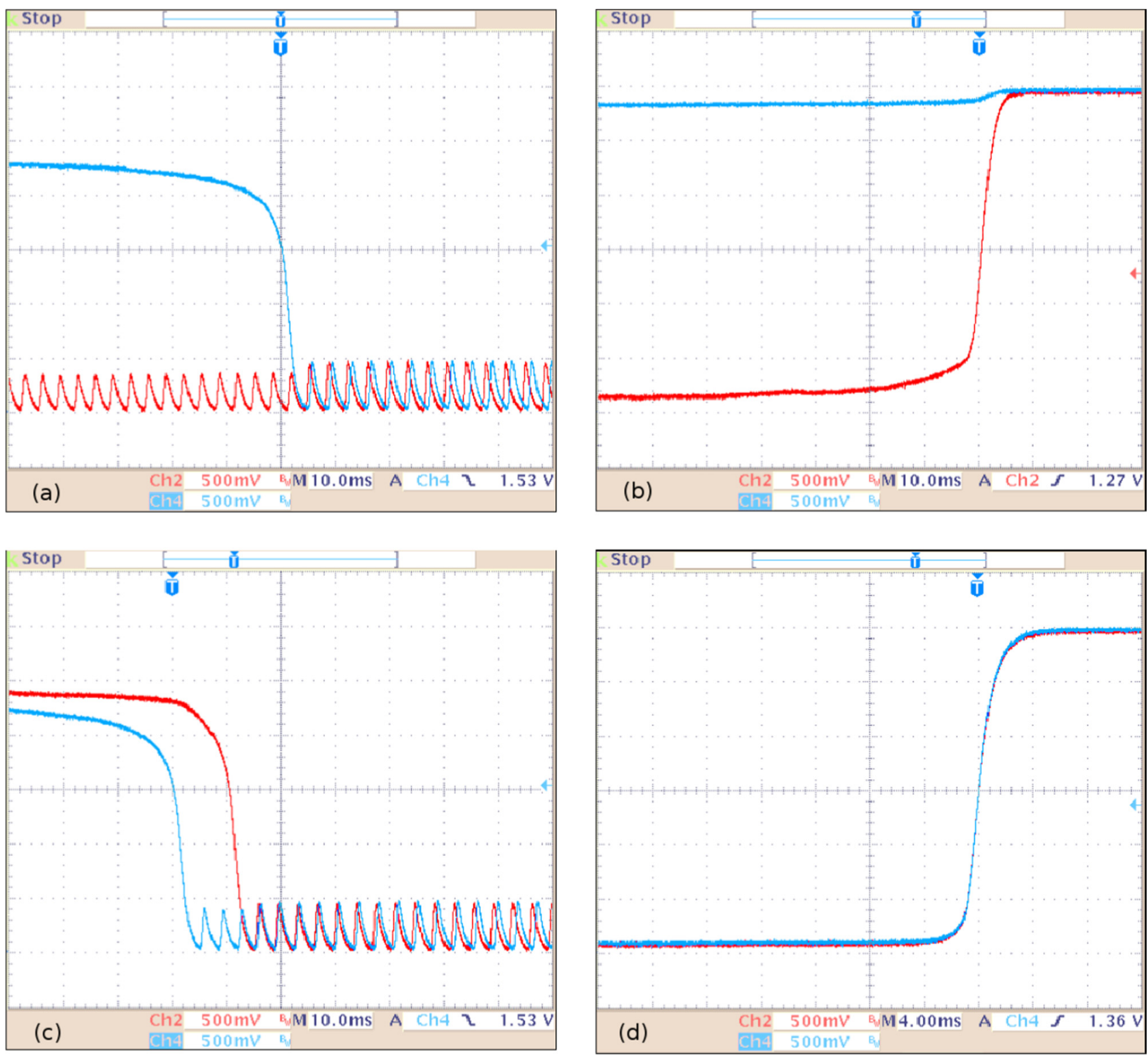

FIG. 5. Oscilloscope screenshots of transitions between stable dynamics in the circuit analog of two QS-coupled repressilators $(n=$ $2.8, \alpha=204, \kappa=17.8$ ). The two traces (blue and red) are the protein $B$ voltages of the repressilators. $Q$ was varied slowly by adjusting a potentiometer. (a) IHLC to APLC for decreasing $Q$ at 0.25. (b) IHSS to HSS for increasing $Q$ at 0.6. (c) HSS to APLC for decreasing $Q$ at 0.15. (d) Low-B-HSS to high-B-HSS for increasing $Q$ at 1.2. Note that in the purely homogeneous case of panel (d), the red trace is not visible because it is covered by the blue trace.

and APLC for $Q$ between LP2 and LP3. This narrow $Q$ range of three-state multistability is embedded within the broader range of two-state multistability. Figure 3(a) displays versatile multistability controlled by coupling strength $Q$.

Figure 3 shows that increasing the activation strength $\kappa$ from 12 to 17 has two qualitative effects. First, the increase produces an IHLC [see time series in Fig. 5(a)] and its associated HB2, which appears where the IHSS becomes unstable between LP2 and LP3. The IHLC extends from HB2 to LP5. Second, the coupling strength $Q$ range for multistability is shifted and increased. For $\kappa=12$, APLC is the only stable behavior for $Q<0.5$, whereas for $\kappa=17$ the regime of multistability extends down to $Q=0.1$, and the $Q$ range for three-state multistability has increased about fourfold. We note that the
LP1 and BP2 points in Fig. 3(b) are too close together to be resolved in the figure, as are LP3 and LP5 for the upper piece of the IHLC. Also, the LP5 of the IHLC extends to a slightly lower $Q$ value than does the LP3 of the unstable portion of the IH branch. The sensitivity of the dynamics in Fig. 3 was investigated by varying parameters $\alpha$ and $\beta_{1}$. Figures 22 and 23 show that these dynamics are not restricted to a narrow parameter range.

Circuit results for $n=2.8, \kappa=17.8$ generally confirm the predictions in Fig. 3(b). Figure 4(a) shows a bifurcation diagram constructed from measured voltage amplitudes for the various dynamics. The APLC branch is stable to $Q=1.2$ where it converts to low-B-HSS, IHLC occurs for $0.25<$ $Q<0.5$, IHSS occurs in $0.5<Q<0.6$, and HSS exists for 


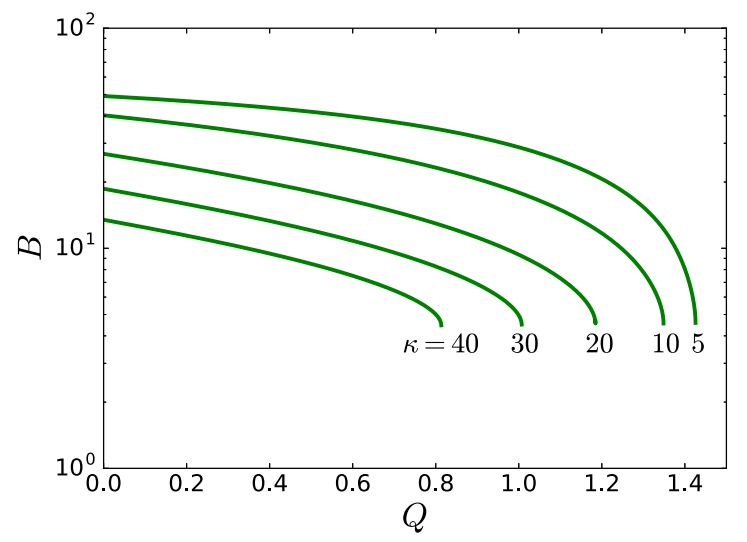

FIG. 6. Numerical APLC branch for various $\kappa . n=2.8, a=204$. Note that for $n=2.8$ the APLC is continuously stable for all $\kappa$.

$Q>0.2$. The low-B-HSS exists over a $Q$ range narrower than the data resolution and therefore appears as a single point at $Q=1.2$. Figures 3 and 4(a) show that for $n=2.8$ APLC is the single homogeneous periodic attractor. Protein $B_{i}$ values in Fig. 4 were calculated from measured voltages as described elsewhere [22].

Measured time series of the dynamics represented in Fig. 3(b) are shown in Figs. 4(b) and 5. Digitized data showing the APLC for $Q=0.4$ is shown in Fig. 4(b). Figure 5 screenshots show all four transitions corresponding to switching between stable branches in the bifurcation diagram as $Q$ crosses limit points: Figure 5(a) shows IHLC to APLC transition for $Q$ decreasing at 0.25 (LP5), Fig. 5(b) shows IHSS to HSS for $Q$ increasing at 0.6 (LP2), Fig. 5(c) shows HSS to APLC for $Q$ decreasing at 0.15 (LP1), and Fig. 5(d) shows low-B-HSS to high-B-HSS for $Q$ increasing at 1.2 (LP4). In addition, the Fig. 5 screenshots show examples of the various dynamics prior to the transitions: IHLC in Fig. 5(a), IHSS in Fig. 5(b), and low-B-HSS in Fig. 5(d). The high-B-HSS is clearly apparent after the transitions in Figs. 5(b) and 5(d). The difference in transition times in Fig. 5(c) indicates that the HSS LP1 is sensitive to unavoidable differences between the two repressilator circuits. We also point out that after the transitions in Figs. 5(a) and 5(c) the phase shifts are in the process of changing to the $180^{\circ}$ characteristic of APLC. $Q$ was slowly varied for the transitions in Fig. 5 by using the potentiometer in the QS circuitry, which sets the value for $Q$ [22].

We note that the $Q$ ranges for particular dynamics in Fig. 4(a) show some differences from the simulation in Fig. 3(b). However, overall agreement of the structure of dynamics is demonstrated. Also, the circuit finds only stable dynamics (and transitions between stable states shown in Fig. 5), and therefore Fig. 4(a) does not find the unstable branches (thin lines) shown in the Fig. 3(b) simulations.

Figure 6 shows simulations of the APLC branch for $n=$ $2.8, \alpha=204$ using various activation strengths $\kappa$. As seen in Fig. 3 increasing $\kappa$ causes suppression of the APLC branch, but not instability.

The dual role of protein $\mathrm{B}$ both directly inhibiting production of $\mathrm{C}$ and indirectly activating $\mathrm{C}$ via $\mathrm{QS}$ is responsible for the expanded regime of high-B-HSS at larger activation

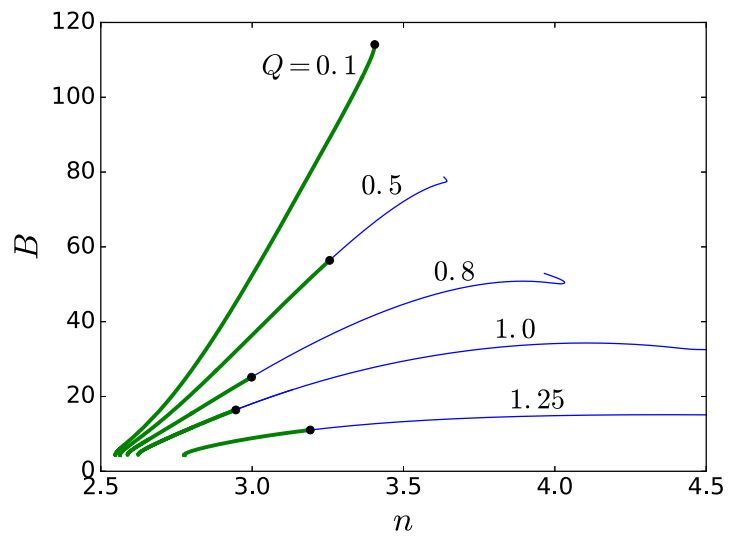

FIG. 7. Stable (thick green) and unstable (thin blue) APLC branch $n$-continuations. Black dots indicate TR (torus) bifurcations where APLC looses stability. $\kappa=15, \alpha=175$.

$\kappa$ seen in Fig. 3(b). At low activation (small $\kappa$ ) and small coupling, each three-gene ring essentially becomes an isolated repressilator and therefore undergoes oscillations with no SS. At large $\kappa$, B's activation of $\mathrm{C}$ overcomes its inhibition and thereby increases expression of protein $C$, which in turn leads to inhibition of $\mathrm{A}$, which results in high-level expression of $\mathrm{B}$ creating a positive feedback for $\mathrm{B}$, in contrast to the negative feedback in an isolated repressilator.

The dynamics demonstrated in Figs. 3-5 both in simulations and in circuits are similar to those found previously for repressively coupled repressilators using similar parameter values [20] and confirmed over large intervals of model parameters. At low coupling strength $Q$ the APLC is the only stable behavior; at higher $Q$ IHLC and then IHSS coexist with both APLC and HSS. In the 14-variable model [20] with $n=2.6$ the APLC branch converts to chaotic behavior when $Q$ reaches 0.6 , whereas for the reduced eight-variable model presented here the APLC branch remains stable. However, as shown below, chaotic behavior is found at higher Hill coefficient $n$.

\section{B. Intermediate repression: $\boldsymbol{n}=\mathbf{3 . 0}$}

The structure of the phase diagrams in Fig. 3 for $n=2.8$ is very rich, and it is interesting to understand its evolution and robustness under the change of such a basic parameter as the Hill cooperativity coefficient $n$. A guide to the interesting $n$-values is provided by the $n$-continuation bifurcation diagram in Fig. 7 for $\alpha=175, \kappa=15$, with coupling strengths $Q=$ $(0.1,0.5,0.8,1,1.25)$. Only the APLC branch is shown since this branch is the sole homogeneous periodic attractor.

Interestingly, the $n$-range of stable APLC (thick green) is restricted between 2.5 and 3.5. Decreasing $n$ below 2.8 reduces the amplitude of the stable APLC. Therefore we increase $n$, expecting the introduction of unstable regimes of APLC (thin blue in Fig. 7) at the larger coupling strengths. Simulations also show that for $n>3.5$ large amplitude APLC is predicted for smaller $\kappa$ at small $Q$ (essentially uncoupled repressilators), and small amplitude APLC is predicted for larger $\kappa$ at large $Q$ ( $\kappa$ suppresses and stabilizes the APLC, shown below).

We begin by increasing repression to $n=3.0$, with $\alpha=190$ and $\kappa=15$. Figure 8 (a) shows the simulated bifurcation 

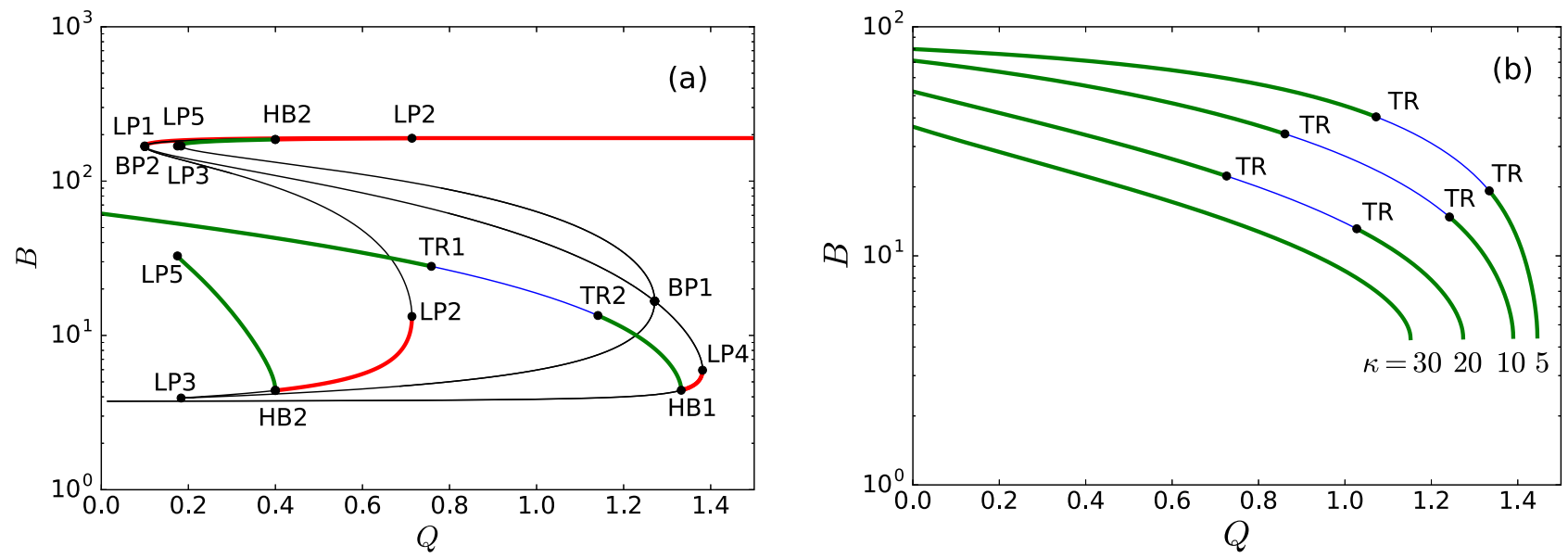

FIG. 8. Numerical $Q$ continuations as in Figs. 3 and 6 for $n=3.0, \alpha=190$. (a) All regimes. $\kappa=15$. (b) APLC for various $\kappa$. Note the region of unstable APLC (thin blue) between the TR bifurcations for the smaller $\kappa$.

diagram. Most of the features are similar to those for the smaller repression case in Fig. 3(b). However increasing repression to $n=3.0$ has introduced the unstable region (thin blue) in the APLC branch between TR1 and TR2 as predicted in Fig. 7. Simulations shown in Fig. 8(b) of the APLC branch for various activation strengths demonstrate how increasing $\kappa$ suppresses and stabilizes the APLC branch, as indicated by the continuous green branch for the largest activation $\kappa=30$.

Figure 9 shows the circuit's measured bifurcation diagram for $n=3.0, \alpha=190, \kappa=17.8$. Note the break in stability in the APLC branch as predicted by the simulations. The similar structure of the dynamics in Figs. 8(a) and 9 demonstrates the agreement of circuit and simulations. Complex oscillations for $n=3.0$ which occur between the TRs are discussed in Sec. D on unstable APLC and chaos.

\section{High repression: $\boldsymbol{n}>\mathbf{3 . 1}$}

Further increase of Hill coefficient $n$ up to 3.2 does not result in qualitative changes in the structure of numerical and experimental bifurcation diagrams although the $Q$ intervals

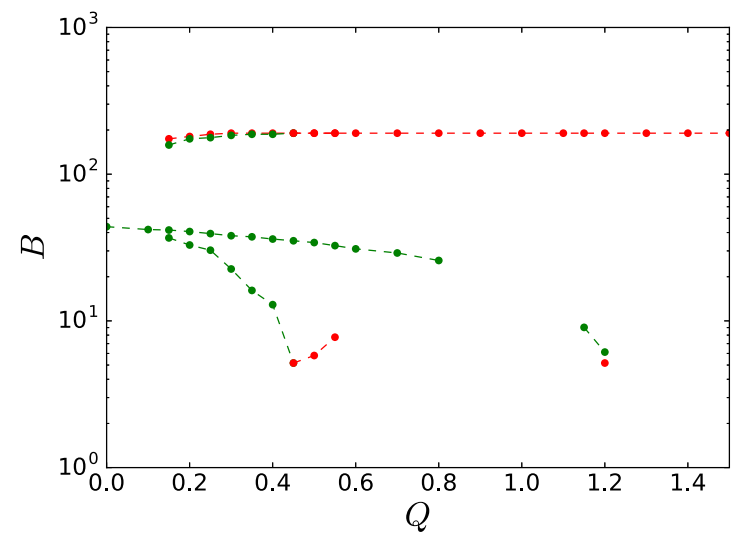

FIG. 9. Circuit $Q$ continuations of all stable branches. $n=$ $3.0, \alpha=190, \kappa=17.8$. Note the break in stability in the APLC branch from $Q=0.8$ to 1.15 . Stable steady state (red), stable limit cycles (green). for IHLC and for chaos are enlarged. In the region of unstable APLC between the TRs a rich variety of behaviors are predicted for $n>3$. The nature of these behaviors is described below in Sec. D.

Increasing Hill coefficient beyond 3.2 eventually leads to the appearance of stable in-phase (IPLC) oscillations: a new dynamic not seen at the smaller $n$-values inside broad intervals of $\alpha$ and $\kappa$. The $n$-continuation diagram in Fig. 10 shows the APLC and IPLC, and the HSS for activation rate $\kappa=4, Q=$ 0.8 , and $\alpha=175$. Stable IPLC occurs in a restricted range of parameters $n, \kappa$, and $Q$. For $\kappa=4, Q=0.8$, the range of repression for stable IPLC is $n=4.12$ to 4.37 . As coupling strength $Q$ is increased (decreased), the $n$-range for stable IPLC moves to lower (higher) $n$-values (results not shown). Then, at a fixed $n$-value there is only a narrow $Q$ range of stable IPLC. For example, at the higher value of $Q=1.0$ the stable IPLC occurs from $n=3.71$ to 3.89 with no overlap of the $n$-range for $Q=0.8$, and at the lower $Q=0.7$ the stable IPLC occurs from $n=4.36$ to 4.8 with overlap only at the upper edge of the $n$-range for $Q=0.8$. The limited $Q$ range of stable IPLC is apparent in the $Q$-continuation diagram in Fig. 11 for $n=4, \kappa=4$. (A linear vertical scale is used in

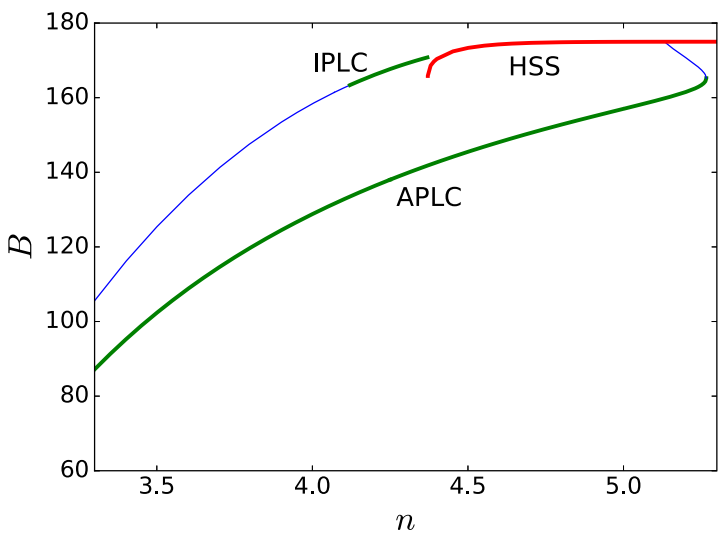

FIG. 10. Numerical $n$-continuation for $\alpha=175, \kappa=4, Q=0.8$ showing coexistence of stable IPLC and stable APLC. Stable LC (thick green), unstable LC (thin blue), and stable HSS (thick red). 


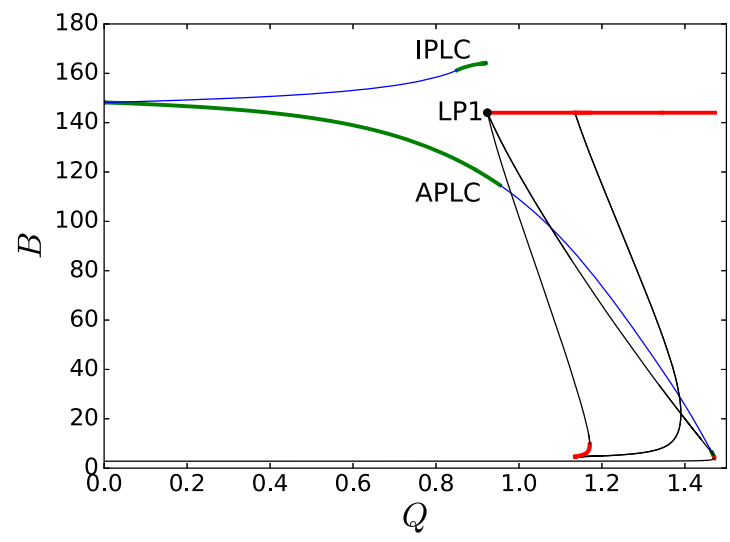

FIG. 11. Numerical $Q$ continuation for $n=4, \alpha=175, \kappa=4$ showing coexistence of stable IPLC and stable APLC. Stable LC (thick green), unstable LC (thin blue), stable SS (thick red), unstable SS (thin black).

Fig. 11 in order to make clear the APLC and IPLC branches.) As $Q$ increases, the IPLC branch ends with an infinite period bifurcation transition to the LP1 of the HSS. Figure 12 shows a screenshot of the opposite transition, from HSS to IPLC, which occurs in the circuit when $Q$ is slowly decreased through the LP1 of the high-B-HSS. Note that the amplitude of the IPLC is larger than the HSS as predicted in Fig. 11.

It was shown above that increasing the rate of activation $\kappa$ causes the LP1 of the HSS to move to lower $Q$ values. This decrease must then also move the end-point of the IPLC branch to lower $Q$ values, which can result in loss of the stable IPLC. For example, raising $\kappa$ to 5 with $Q=0.8$ causes the $n$-range for stable IPLC to move to $n=3.8$ and shrink to only 0.008 width (compared to width 0.25 at $n=4.25$ for $\kappa=4$ in Fig. 10), and it causes the complete loss of stable IPLC for all

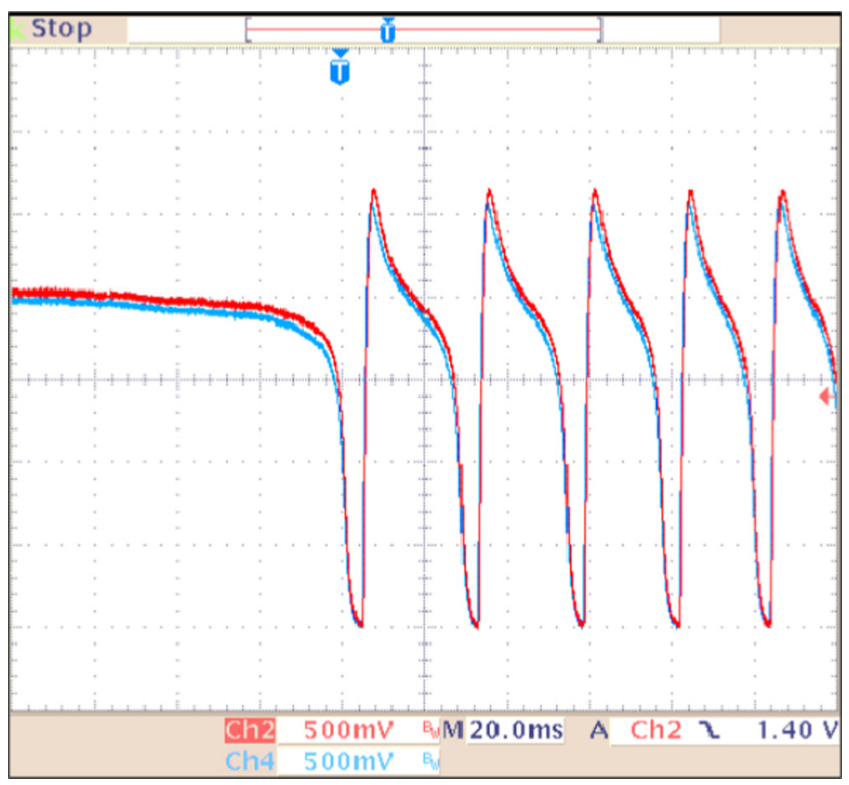

FIG. 12. Time series demonstrates transition from HSS to IPLC in circuit for decreasing $Q$ at 0.85 (LP1); $n=4, \alpha=182, \kappa=4.2$. The two traces (blue, red) for the protein $\mathrm{B}$ voltages of the two repressilators are nearly identical. $n$-values for $Q>0.8$. The $Q$ interval for stable IPLC can be made longer by increasing $\alpha$ (data not shown). Appearance of the stable IPLC is the only new stable attractor at the larger cooperativity of repression. The IPLC coexists with stable APLC, which remains the dominant dynamical behavior.

\section{Unstable APLC regime, quasiperiodicity, and chaos}

In the region of unstable APLC between the TRs [see Figs. 8(a) and 9] a rich variety of behaviors are predicted numerically and observed in the circuit for $n \approx 3.0-3.4$. Figure 13 shows screenshots for the stable APLC region (at $Q=0.6$ ) and the unstable region (at $Q=0.9$ ) in the circuit for the Hill coefficient $n=3.0$. The nature of the dynamics was investigated by using the oscilloscope's built-in fast Fourier transform (FFT) function. Figure 13(a) shows stable APLC oscillations in the upper portion as indicated by distinct peaks in the FFT in the lower portion. The DC component peak is seen at the left edge and peaks for the fundamental (at $270 \mathrm{~Hz}$ ) and the second, third, and fourth harmonics are clearly apparent. Figure 13(b) shows complex oscillations (COs) in the upper portion and a continuum FFT in the lower portion, which suggests that oscillations are possibly chaotic. The COs begin with the appearance of subharmonic peaks in the FFT for $Q$ just beyond TR 1 bifurcation ( $Q=0.8$ ). The continuum FFT predominates for intermediate $Q$ values, followed by the return of subharmonic peaks when $Q$ approaches TR2 $(Q=1.1)$ where a transition to the small-amplitude APLC occurs. No transitions to HSS were observed in circuits for $Q$ values varied gradually between the TRs for $n=3.0$.

Simulations with $n=3.0, \alpha=190, \kappa=15$, and $Q$ ranging from 0.75 to 1.10 reveal that COs are the dominant behavior between the TRs and that there are no transitions to HSS, even with the addition of intensive white noise. The $\mathrm{CO}$ regime persists, as observed in the circuit although there may be differences in the nature of the $\mathrm{CO}$ attractor depending on the values of coupling strength and $\alpha$. COs can lose stability within certain $Q$ ranges, whereas clear evidence of chaotization (based on the distribution of recurrence times as well as their sequential maps; see Appendix B) are observed in others. The detailed study of $\mathrm{CO}$ evolution is not our goal in this work, and we use this designation as a general term, keeping the term "chaos" only for when we are sure about the chaotic character of the dynamics.

The main dynamical surprise found between TRs for $n=3.0$ over a large range of parameter $\alpha(180$ to 300$)$ is the coexistence of the $\mathrm{CO}$ regime and periodic limit cycle with five subperiods ("return times") labeled 5:5LC. Figure 14 presents the phase portraits of 5:5LC and COs calculated using identical parameters, but different initial conditions. It is clearly seen that 5:5LC and this type of $\mathrm{CO}$ are antiphase spatially symmetric attractors similar in structure and slightly different in amplitudes. Figure 15 presents the distributions of return times of the coexisting attractors, calculated using Poincaré sections $\left(B_{1}=B_{2}=5\right)$ of very long trajectories in the presence of uncorrelated low-level white noise added to variables $B_{i}$. Integrations were started from initial conditions corresponding to 5:5LC [Fig. 15(a)] or to COs [Fig. 15(b)]. Noise amplitudes were varied to find the most appropriate value to escape the endless and fast mixing of dynamic 

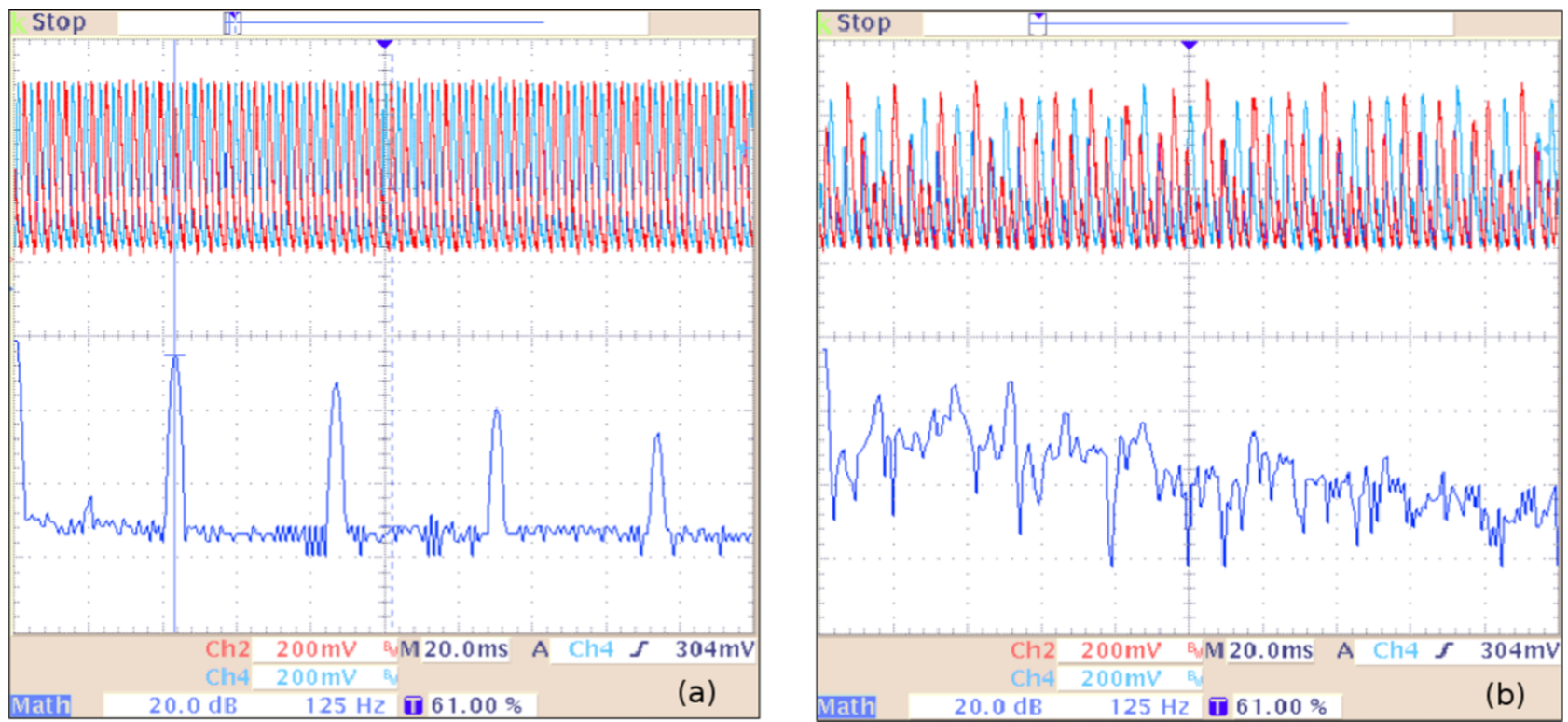

FIG. 13. Oscilloscope screenshots of time series and FFT demonstrating (a) stable APLC with distinct frequencies in FFT at $Q=0.6$ and (b) CO with continuum FFT at $Q=0.9$. Protein B voltage traces (blue, red) in top portion, FFT of red trace in bottom portion. $n=3.0, \alpha=190, \kappa=17.8$. Stable APLC returns for $Q>1.1$ (see Fig. 9).

regimes. There is an interval of noise amplitudes which stimulates the appearance of visible dispersion of all five peaks [corresponding to the return times in Fig. 15(a)] in $d N / d T$ distributions, but it is too small to stimulate switching between the periodic 5:5LC and the COs. This means that despite similarities in the extent of phase relations (Fig. 14) and in the boundaries of return times (Fig. 15), the system Eq. (1) has two types of coexisting stable solutions-5:5LC and $\mathrm{CO}-$ over large intervals of parameters inside the region of unstable APLC.

Figure 16 shows two qualitatively different one-parameter continuations of 5:5LC which demonstrate the basic bifurcations controlling the dynamics of the system. For a particular set of parameters $(\alpha=200, \kappa=15)$ the $\mathrm{CO}$ regime occupies the entire $Q$ region between TR bifurcations at 0.74 and 1.14 (not shown) thereby coexisting with the entire 5:5LC bounded by LP bifurcations at 0.879 and 1.03. The COs demonstrate quasiperiodic behavior after the lower TR up to the lower LP where stable 5:5LC emerges. In the region of their coexistence narrow periodic windows appear in the COs indicating a
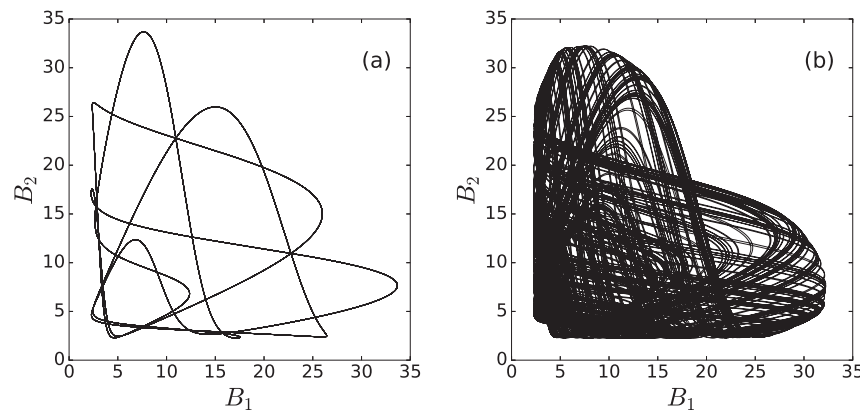

FIG. 14. Phase plots of coexisting dynamics for two QS-coupled repressilators $(n=3.0, \alpha=200, \kappa=15, Q=0.935)$. (a) 5:5 limit cycle. (b) Complex oscillations. Initial conditions are different for the two panels. change in the CO dynamics. At the higher value of $\alpha=270$ both attractors have lost continuity, and a pitch-fork bifurcation (BP) has introduced a slightly asymmetric branch of the 5:5LC. The 5:5LC loses stable continuity due to a period-doubling cascade to chaos just beyond the lower PD and regains stability via a period-halving cascade at the upper PD. The regions of stable COs for $\alpha=270$ are marked by violet lines ending in circles, resulting in coexistence with 5:5LC just near the LPs. Chaos born from PD cascades is the sole oscillating attractor over the broad $Q$ range between PDs. For $\alpha<295$ the chaos attractor remains separated from the $\mathrm{CO}$ attractor by $Q$ regions where 5:5/As5:5LC is the sole oscillating attractor.

We use the notation Asn:mLC to indicate asymmetric LCs located inside the unstable APLC region between the TR bifurcations. These LCs are either bounded by limit points or emerge from pitch-fork bifurcation of limit cycles, thereby distinguishing them from the IHLCs which emerge from Hopf-bifurcation of inhomogeneous steady-state solutions and are independent of chaos formation.

In summary, raising the Hill coefficient to $n=3$ opens new complex dynamic behaviors which are different from classic chaos found earlier for two coupled repressilators $[19,20]$ : the coexistence of the $\mathrm{CO}$ and 5:5LC attractors for $\alpha \approx 200$; then for increasing $\alpha$, the loss of stable COs over a $Q$ range, followed at higher $\alpha$ by the loss of stable 5:5LC over a $Q$ range and its replacement by chaos born from PD cascades. The chaos attractor is distinct in character from the $\mathrm{CO}$ attractor, and they are separated by $Q$ regions where 5:5/As5:5LC is the sole oscillating attractor over a large $\alpha$-parameter range. For the investigated broad areas of model parameters 5:5LC is "isola" in terms of bifurcation theory, not linked to other regimes in the investigated ranges of parameters. Bifurcation analysis of its evolution as a function of $Q$ and $\alpha$, its competition with COs, as well as its transformation into chaos in parameter space deserves special investigations beyond the scope of this paper (work in progress). 

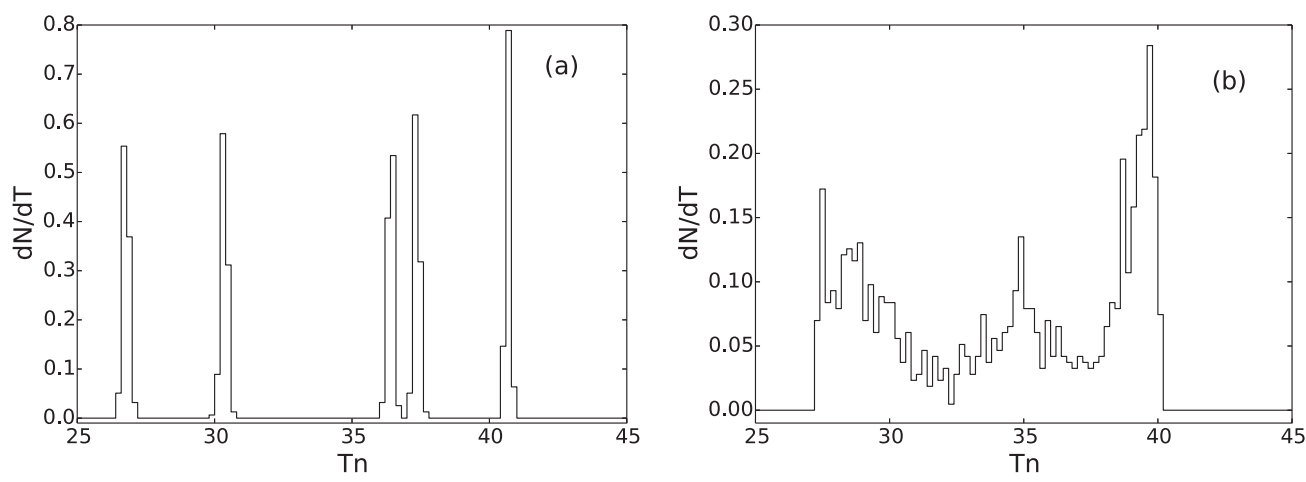

FIG. 15. Distributions of return times collected from the Poincaré sections of trajectories of 5:5LC (a) and CO (b) in the presence of small noise. $n=3.0, \alpha=200, \kappa=15, Q=0.935$.

Next we consider a higher repression, $n=3.15$ to 3.2 , which leads to new qualitative changes in the dynamics in the region between TR bifurcations despite the small increase in the Hill cooperativity coefficient. By way of example, we show time series of some new dynamics from both simulations and circuit measurements, then we describe in detail the $Q$-continuation progression of the unstable APLC-branch dynamics for a representative case of $n=3.15, \alpha=225$, and $\kappa=10$. Additional examples of behaviors from simulation and circuit measurement are shown in the Appendix B.

The basic regular attractors between TR bifurcations are high-period symmetric limit cycles n:nLC and a set of spatially asymmetric limit cycles. Numerical simulations of stable 3:3LC and As1:2LC behaviors are shown in Figs. 17(a) and 17(b), and their observations in circuit are shown in Figs. 17(c) and 17(d). In the circuit we search for unstable APLC behaviors by varying component values, with the understanding that no set of values gives a perfect match to the Hill function repression for a particular value of $n$. Therefore when looking for similar behaviors in simulation and circuit measurement as in Fig. 17 we are not concerned with exact matches of parameter values $(n, \alpha, \kappa)$ between circuit and simulation.

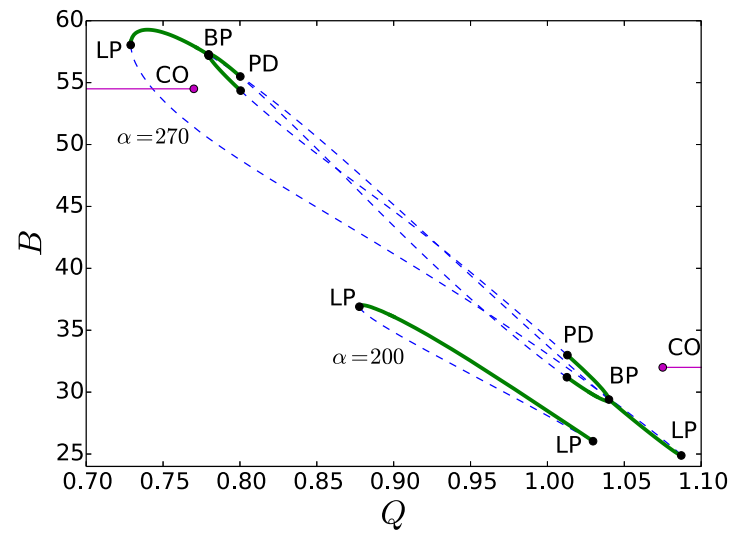

FIG. 16. Q-continuations of 5:5LC, stable (solid) and unstable (dashed), for $\alpha=200$ and $270, n=3, \kappa=15$. For $\alpha=200$ the entire 5:5LC coexists with complex oscillations (COs). For $\alpha=270$ the violet lines ending at small circles indicate regions of COs. BP, branch point (broken symmetry bifurcation); $\mathrm{PD}$, period doubling bifurcation; LP, limit point.
Figure 18 shows the $Q$ continuation of several attractors over the region where APLC is unstable. The basic regime branches - stable APLC, IHSS, and HSS-are familiar from previous diagrams as are the torus bifurcations TR1 and TR2, and the Hopf bifurcation HB1. However, Fig. 18 shows many new regimes in multistable dynamics of system Eq. (1): high-period limit cycles for 3:3LC [similar to Fig. 17(a) for $n=3.19$ ], 6:6LC, and spatially asymmetric limit cycles [similar to Fig. 17(b)]. Unstable portions are shown with broken lines for clarity. Four of the period doubling points are shown. Also shown are five narrow windows with stable attractors: As4:4LC from $\operatorname{LP}(Q=0.710)$ to $\operatorname{LP}(Q=0.719)$, the stable ends of a 5:5LC at $\operatorname{LP}(Q=0.712)$ and $\operatorname{LP}(Q=$ 1.247), and the stable ends of an $\operatorname{As} 4: 4 \operatorname{LC}$ at $\operatorname{LP}(Q=0.727)$ and $\operatorname{LP}(Q=1.268)$ (very close to $3: 3 \mathrm{LC}$ ). The unstable portions of the 5:5LC and the As4:4LC are not shown in Fig. 18 due to confusing overlap with other branches. (The 5:5LC may be the evolution of the 5:5LC shown above for $n=3.0$.) Many other narrow windows with stable symmetric and asymmetric high-period LCs also exist but are not shown. The numerous coexisting dynamical branches have a strong influence on the nature of the $\mathrm{CO}$ inside the region of unstable APLC. We use Fig. 18 as a reference guide as we describe simulation results found using fixed values of coupling strength $Q$. Note that Fig. 18 zooms in to make clear the various dynamic branches and therefore does not show the high-B-HSS which is apparent in previous Figs. 3(a) and 8(a). The stable high-B-HSS competes with the other attractors for phase space over the entire region of unstable APLC.

Similar to the case of $n=3$, the first regime after the TR1 bifurcation is a quasiperiodic attractor characterized by "beating" of the APLC time series producing two peaks in return times distributions. With further increase of $Q$, a third peak emerges suggesting the approach of chaos (all within the broken violet line in Fig. 18 from TR1 to 0.735). The more effective indicator of chaos is the emergence of a strongly folded sequential period map presented in Fig. 24 to demonstrate the process of chaos maturation as $Q$ grows.

Within the region between TR1 and $Q=0.735$ there are many very narrow periodic windows which may be occupied by amplitude-symmetrical LCs (e.g., 13:13LC, 35:35LC, and other n:n-LCs) or by slightly amplitude-asymmetrical regimes (e.g., the As4:4LC). Many of these regimes in narrow windows demonstrate period doubling bifurcations returning system to 

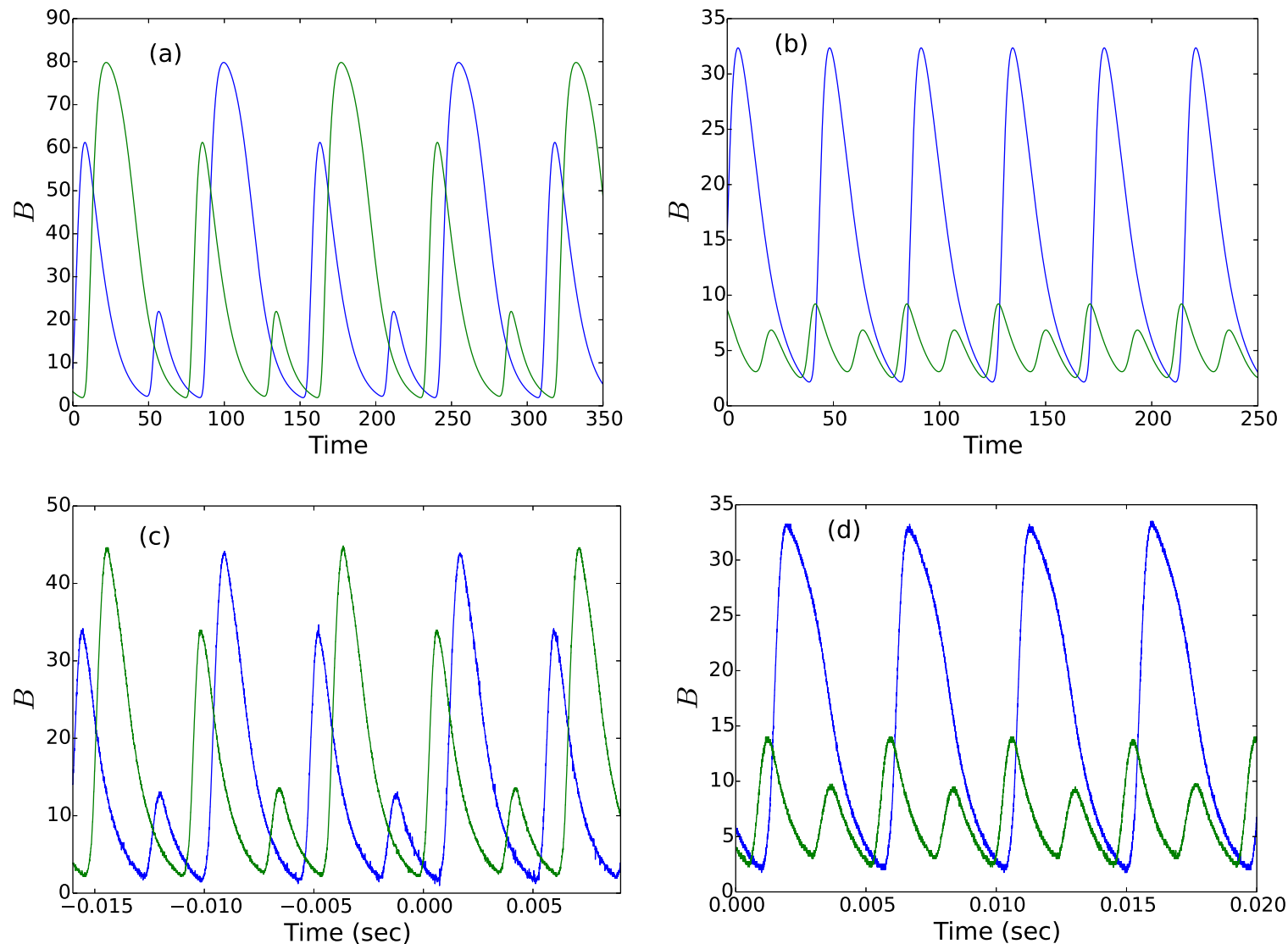

FIG. 17. Time series comparing the same LC dynamics in numerical simulations (a, b) and in circuit measurements (c, d). (a) 3:3LC numerical. $n=3.19, \kappa=15, Q=0.6$. (b) As1:2LC numerical. $n=3.2, \kappa=15, Q=1.092$. (c) $3: 3 \mathrm{LC}$ measured. $n=3.2, \kappa=7.3, Q=1.05$. (d) As1:2LC measured. $V_{t h}=16 \mathrm{mV}$ and $R_{\text {hill }}=2.9 \mathrm{k} \Omega$.

chaos. For example, the 5:5LC starts at 0.7116 as a stable symmetric 5:5LC which branches to slightly asymmetric 5:5LC at 0.7141 and then starts a PD cascade to chaos at 0.7152 . This regime coexists with asymmetrical $4: 4 \mathrm{LC}$

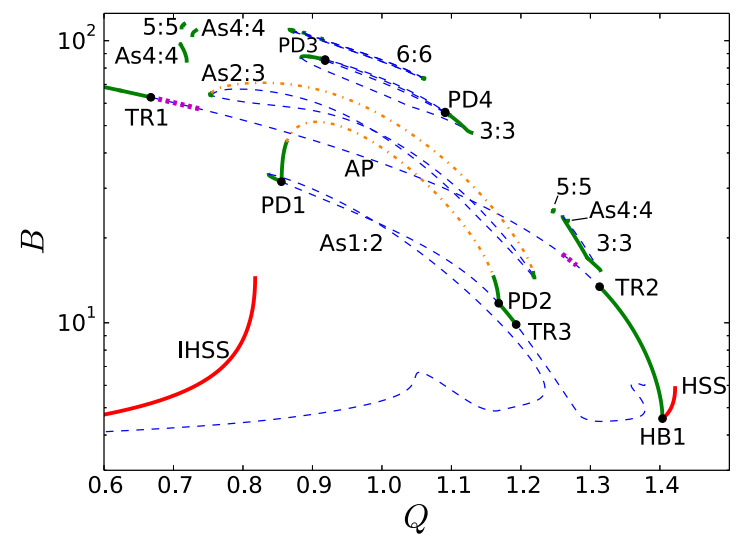

FIG. 18. $Q$ continuations of stable (green) and unstable (dashed) LC, and stable SS (red) for $n=3.15, \alpha=225, \kappa=10$. Narrower windows of stable high-period LCs not shown. For clarity only the lower amplitude oscillation of asymmetric LCs is shown. The first period-doubled branches for As1:2 and As2:3 are shown (stable, green; unstable, orange dot-dash). Regions of "simple" antiphase complex oscillations (broken violet) exist near the torus bifurcations (TR) of the APLC branch. (see their phase portraits and zoom-in $Q$ continuations in Figs. 26 and 27). In contrast to the 5:5LC, the As 4:4LC from $Q=0.710$ to 0.719 is "isola" because its $Q$ interval is limited by LP bifurcations. The chaos emerged through the region of TR1 to 0.735 is characterized as a "simple" antiphase attractor similar to that presented above in Fig. 14(b) for $n=3$, which means that elements of the highly asymmetric behavior arise only for $Q>0.74$.

Spontaneous durations of large amplitude asymmetric rhythmic behavior, which is our main target here, begin to appear within chaos for $Q$ beyond 0.74 thus ending the region of simple chaos. These AsLC durations are related to the appearance of AsLC branches extending over a broad $Q$ range which have stable regimes near their low- $Q$ and high- $Q$ limit points. For example, Fig. 18 shows continuations As2:3LC (low- $L P=0.7528$ ) and As $1: 2 \mathrm{LC}$ (low- $L P=0.837$ ) extending to the high-LP (1.219 for As2:3LC) and to TR3 (1.193 for As 1:2LC). Various stable n:n regimes also appear including portions of the $6: 6 \mathrm{LC}, 3: 3 \mathrm{LC}$, and 5:5LC. In contrast to the dynamics for $n=3.0$, there are no broad $Q$ intervals with the coexistence of chaos and stable limit cycles. The stable regimes (for both AsLC and n:n-LC) typically undergo period-doubling cascades to chaos at the low- $Q$ end of their branch, and period-halving cascades (for increasing $Q$ ) from chaos back to the stable high- $Q$ portion of the branch. The coexistence of numerous dynamical branches, each with intervals of stability and cascades to chaos, has a dramatic effect on the types of 

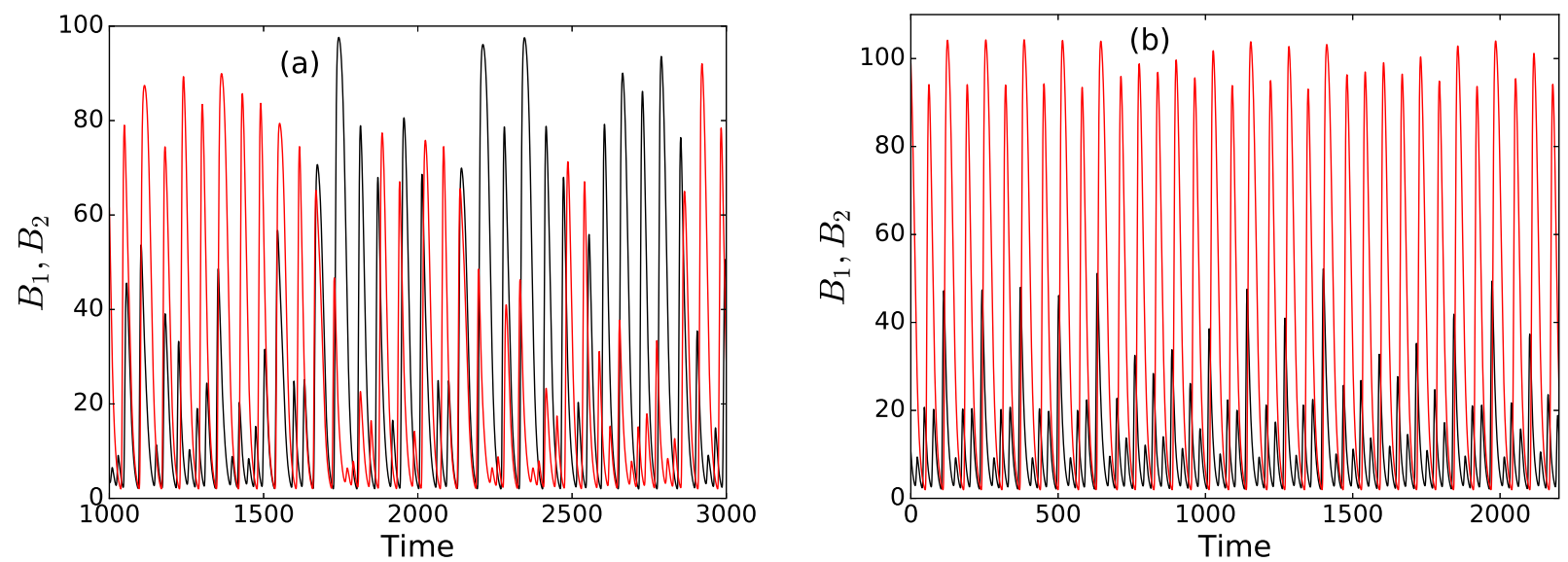

FIG. 19. Numerical time series of chaotic attractors for QS-coupled repressilators $(n=3.15, \alpha=225, \kappa=10)$. (a) Branch switching of the repressilators resulting in symmetric chaos, $Q=0.95$. (b) As chaos as a result of no switching, $Q=0.87$.

chaos manifestations. Much of the chaos between $Q=0.74$ and 1.25 consists of switching between pieces of the various coexisting unstable branches. In particular, many pieces of As 1:2LC are found in the chaotic trajectory over the $Q$ interval spanned by the As1:2-LC branch. A typical example of time series observed for value $Q=0.95$, which is chosen far from the boundaries of periodic windows to exclude the overlay with intermittency, is presented in Fig. 19(a) (other examples shown in Figs. 28 and 29). It contains the alternating parts of asymmetric attractors as well as the elements of 3:3LC.

The stable As1:2LC and As2:3LC both convert to purely asymmetric chaos (As chaos) via period-doubling cascades (PD points for As2:3LC are not shown since they would obscure the small regions of stable LC). The first PD branches of As1:2LC and As2:3LC are shown using orange broken lines for their unstable portions. The stable As1:2LC extends from a limit point at 0.837 to PD1(0.855) and from PD2(1.168) to TR3(1.193). The period-doubled stable As2:4LC extends from PD1 to 0.8627 and from 1.1609 to PD2. The As chaos (located near the ends of the unstable PD branches in orange) exists over a narrow $Q$ interval beyond which it typically converts to the chaos consisting of switching between pieces of coexisting unstable branch dynamics. The largest regime of purely asymmetric chaos (with no switching) occurs at the end of the PD1 cascade. This cascade (As2:4LC at 0.855, As4:8LC at 0.8627) of As1:2LC ends in asymmetric chaos [Fig. 19(b)], which coexists with the stable 6:6LC, with both the asymmetric chaos and the stable 6:6LC ending at $Q=0.872$. The asymmetric chaos is distinct from the chaos composed of pieces of asymmetric behavior, which over time is symmetric due to the chaotic switching of oscillators amplitudes [Fig. 19(a)].

The existence of different nearby stable attractors determines the creation of intermittent behaviors composed from different combinations of unstable attractors. Typical examples of intermittency localized near the boundaries of periodic windows are exhibited in Figs. 30 and 31, but their analysis is beyond the scope of this paper.

Beyond $Q \approx 1.25$ the number of coexisting dynamical branches is reduced, causing the chaotic behavior to change character (indicated by the broken violet line in Fig. 18). The chaos is characterized by pieces of symmetric
n:n-LC behavior and contains none of the highly asymmetric behavior associated with the As 1:2LC and As2:3LC branches. (Apparently the unstable As1:2LC portion beyond TR3 does not contribute to the chaotic behavior.) The chaos coexists with the stable $3: 3 \mathrm{LC}$ out to about 1.28 . For $Q$ from 1.28 to TR2 the stable 3:3LC and the HSS capture all the behavior. "Beating" exists just below TR2, occurring at $Q=1.3104$ and converting to $3: 3 \mathrm{LC}$ at $Q=1.3102$ and is mentioned here only to show the qualitative similarity of dynamics near TR1 and TR2 bifurcations.

Finally, we consider the unstable APLC region for $n$ beyond 3.2. Generally, simulations find that parameters $\alpha$ or $\kappa$ have to be changed in order to find interesting regimes (chaos, asymmetric limit cycles, symmetric regular n:n-LC, intermittency) like those demonstrated for $n$ around 3.15. For example, for $n=3.3$ and $\kappa=15$ the interesting regimes appear only if $\alpha<120$. In contrast, for $\kappa=4$ the system demonstrates only stable APLC for $100<\alpha<200$; however, the typical complex evolution $Q(T R 1) \rightarrow 5: 5 \rightarrow 4: 4 \rightarrow 1: 2 \rightarrow 3: 3$ is restored when $\alpha=250$ with $Q>0.945$. Hill coefficient $n=$ 3.5 with $\kappa=15$ requires further reduction in $\alpha$ (55-70) for the existence of interesting multistability, which are then located at $0.7<Q<1.1$. If activation is significantly reduced $(\kappa=4)$, then $\alpha$ must be increased to get interesting regimes. However, these regimes are then located at $Q>1$ if $100<\alpha<180$. We conclude that limited but significant variations of basic parameters in 3-dim space do not discover other qualitatively new dynamic regimes compared to those described above.

\section{DISCUSSION}

Using simple four-dimensional mathematical and electronic models of identical ring oscillators we demonstrated that the realistic design of quorum sensing coupling proposed earlier [20] leads to remarkable multistability even for a pair of oscillators. A QS-coupling system can be incorporated into genetic circuits in different ways, acting solely as a coupling agent (as in the case of repressilators [11] and relaxators [10]), or it may also be an integral part of an individual oscillator $[12,13,27]$. In any case, the additional differential equation for autoinducer contains a term describing its linear diffusion, but the resulting coupling is nonlinear 


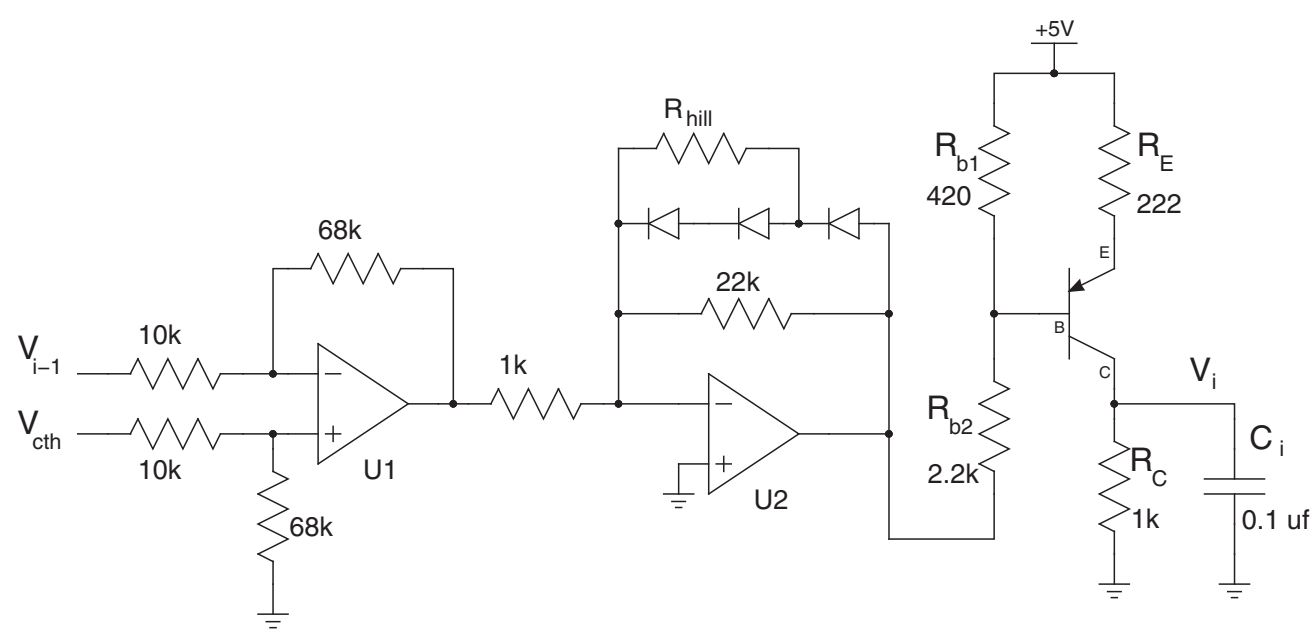

FIG. 20. Electronic circuit used to model a single gene in the QS-coupled repressilator circuit. Output $V_{i}$ is the concentration of expressed protein, and $V_{i-1}$ is the inhibitor concentration. Circuit parameters $V_{\text {cth }}$ and $R_{\text {hill }}$ are adjusted to obtain the desired Hill function inhibition.

for the oscillators because of a delay in the autoinducer production and because the production of one of the oscillator's variables is activated nonlinearly. Therefore it is natural to expect unusual collective modes for QS-coupled oscillators, as was demonstrated in Ref. [20]. However, that study of multistability considered mainly the role of coupling strength, with the other parameters of the single oscillator like repression cooperativity and the transcription rate being fixed. Therefore, the generality of QS-induced multistability was still obscure because the origin of many new regimes can be traced to modulations in oscillation amplitudes and the "steepness" of repression which are strongly dependent on the dynamic properties of the isolated oscillators.

We extended the study by addressing the roles of the other parameters and detected an interesting evolution of multistability in the three-dimensional parametric space defined by Hill cooperativity coefficient $(n)$, AI-induced activation $(\kappa)$, and the rate of transcription $(\alpha)$. This extension is not a pure theoretical exercise; rather, it is stimulated by experimental progress in the development of engineered promoters having different strengths and structures that control the cooperativity in binding of transcription factors.

Combination of numerical simulations, bifurcation analysis, and electronic measurements is beneficial to investigations of dynamical systems like the synthetic genetic network studied here. Benefits come from identifying the similarities and the differences between results of the numerical and electronic models. Dynamic characteristics that are similar in both systems are unlikely to be particularly sensitive to the model parameters and, therefore, could be present in other similarly constructed systems, possibly even real biological systems in the future.

For small $n$ around 2.8 our reduced four-dimensional repressilator models reproduce the basic regimes found in the seven-dimensional model used in Ref. [20]: stable APLC started for small and ended at high coupling strength and stable homogeneous and inhomogeneous steady states, as well as inhomogeneous limit cycle at intermediate coupling strengths. Multiple regimes may coexist over a broad range of control parameters.
Further smooth increase in Hill coefficient $n$ in association with the respective adaptation of activation $\kappa$ introduces an unstable APLC via torus bifurcation over a region of coupling strengths as demonstrated by the results for $n=3.0$. With increasing coupling strength, the dynamics in the region where the APLC is unstable shows gradually developing complex oscillations (as can be detected using FFT analysis and/or from calculations of subperiod distributions), which begin with beating near the TR bifurcations. An extraordinary property of the dynamics for $n=3$ is the coexistence of the developing complex oscillations and the stable limit cycle consisting of five subperiods (see Figs. 14 and 16), which itself is an isolated solution. The $Q$ intervals of coexistence strongly depend on the amplitudes of isolated repressilators, being very long for $\alpha=200-220$ and nearly negligible for $\alpha>350$. Starting from intermediate $\alpha$ ( $\alpha>250$ for our parameter set) the emergence of chaos at low $Q$ and its extinction at higher $Q$ are the result of sequential branch point and period doubling bifurcations of this 5:5LC. Each local bifurcation of our system on the $(\alpha, Q)$ plane for $n=3$ is well known but the structure of the map of regimes as a whole is unusual to the best of our knowledge and is a subject of further studies.

We found the interval of $n$-values where chaos in two QScoupled repressilators is not an exotic regime but instead exists in a large three-dimensional parametric space. Moreover, a further increase in $n$ up to $n=3.15$ introduces new behaviors inside the $Q$ interval with the unstable regimes, including regular and irregular asymmetrical ones (Figs. 17 and 19). All collective modes presented in our study are the result

TABLE I. Model parameters and circuit values.

\begin{tabular}{lccc}
\hline \hline$n$ & $\alpha$ & $V_{t h}(\mathrm{mV})$ & $R_{\text {hill }}(\mathrm{k} \Omega)$ \\
\hline 2.8 & 204 & 14.5 & 2.5 \\
3.0 & 190 & 15.5 & 2.7 \\
3.1 & 184 & 16.0 & 2.9 \\
3.17 & 218 & 13.5 & 4.0 \\
3.2 & 178 & 16.5 & 2.9 \\
4.0 & 182 & 16.2 & 9.0 \\
\hline \hline
\end{tabular}




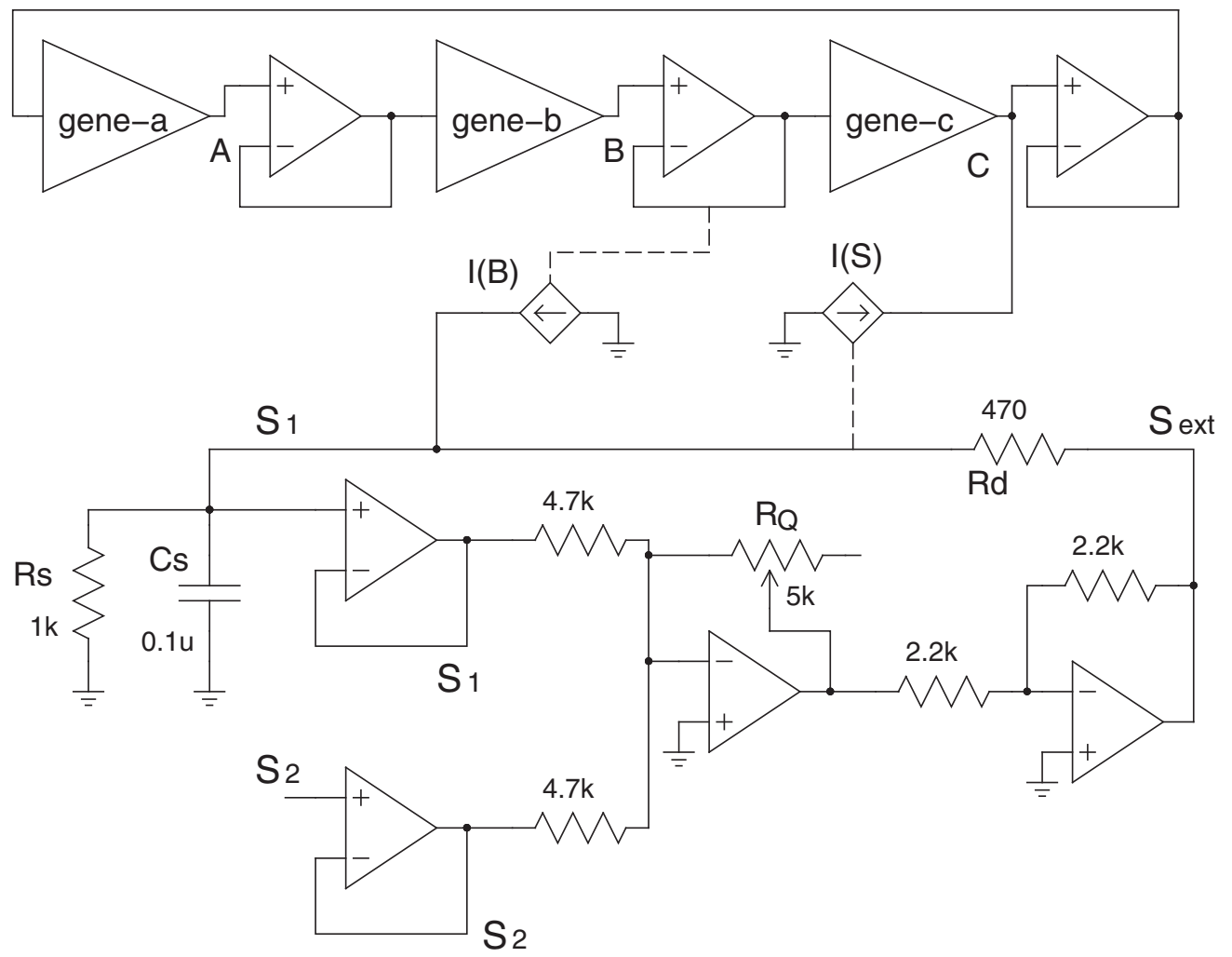

FIG. 21. Circuit for repressilator with QS feedback. The repressilator consists of the closed ring of genes A, B, and C. The quorum sensing loop is from $B$ through $S_{1}$ to $C$. Protein B creates autoinducer $S_{1}$ via the voltage-controlled current source $I(B)$, and $S_{1}$ activates production of C via $I(S)$. Each "gene" triangle corresponds to the single gene circuit in Fig. 20. $S_{2}$ is the contribution from a second repressilator (not shown), and $S_{\text {ext }}$ is the autoinducer concentration in the external medium. Coupling strength $Q$ is controlled by resistor $R_{Q}$.

of competition between repression [Fig. 2(a)] and activation [Fig. 2(b)] of transcription of one specifically selected gene. These antagonistic impacts have different dependencies on the values of the variables: repression is cooperative while activation is linear up to saturation. This means that variation of one parameter, e.g., Hill coefficient $n$, may require the other parameters be tuned to escape the transition of the system to a simple attractor like HSS.

We investigated several sets of parameters and found that the evolutions of the complex regimes as a function of coupling strength are qualitatively very similar. Then we looked at the typical dynamics of chaos and discovered its basic skeleton although many details are still unclear. The most impressive result is the existence of several types of chaos: (1) simple antiphase chaos riddled with very narrow periodic windows of symmetric regimes of the n:n-LC type; (2) asymmetric chaos resulting from period doubling of regular asymmetric regimes, e.g., As 1:2LC and As2:3LC; and (3) symmetric chaos consisting of symmetric and asymmetric parts with alternating polarities. The last type of chaos is the most flexible in its
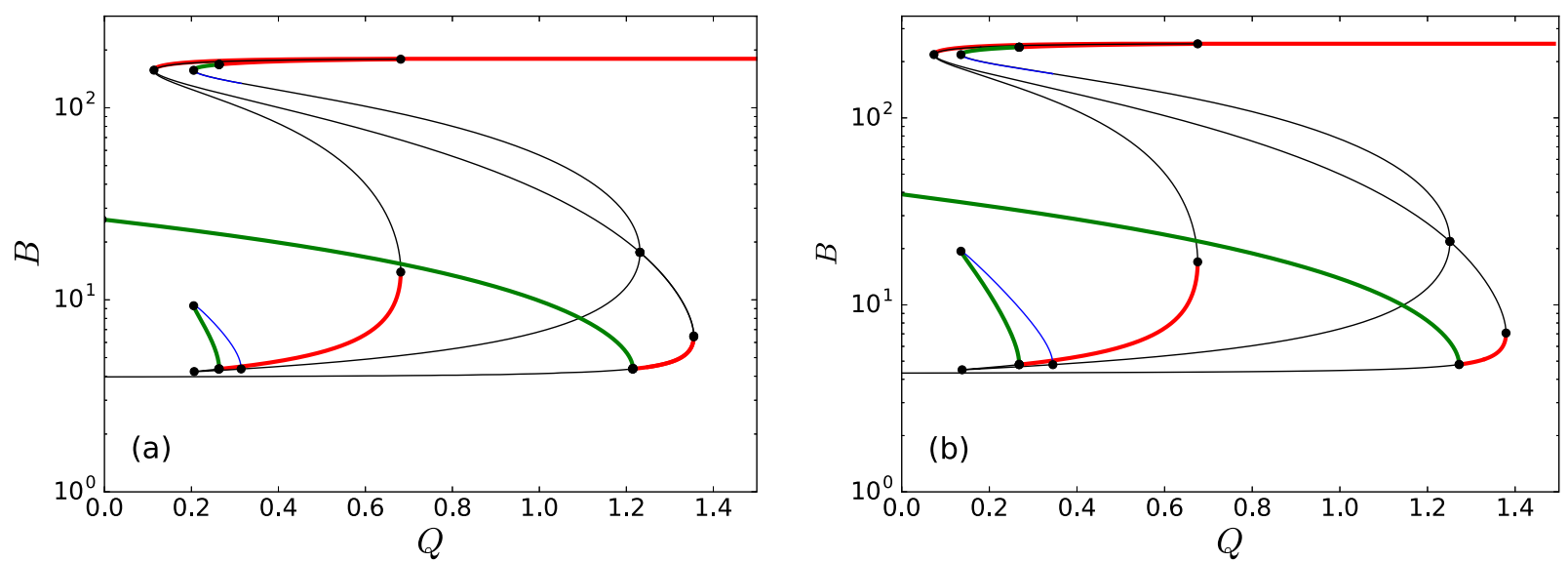

FIG. 22. Numerical $Q$ continuations for $n=2.8$ demonstrating robustness of dynamics over wide range of $\alpha$. Compare with Fig. 3 . (a) $\alpha=180, \kappa=18$. (b) $\alpha=250, \kappa=15$. Solid thick (thin) lines are stable (unstable) attractors. 
specific manifestations and is closely linked to intermittency, which is also observed but only near the boundaries between the regimes.

The possibility for chaos due to linear diffusion between identical regular oscillators is known since the middle of the 1970s [28-31], but the parameter regions of chaos are typically very narrow. Chaos via torus bifurcation of APLC has been observed in two Rössler oscillators coupled by crossdiffusion [32], in two coupled neurons [33,34], and in two nonlinearly coupled biochemical oscillators [35]. To the best of our knowledge there is only one example of chaotization due to nonlinear coupling of identical biochemical oscillators where "bichaoticity" was demonstrated in narrow parameter intervals without analysis of other regimes inside chaos [36].

A large cooperativity $n$ (around 4) opens the gate in parameter space for the in-phase limit cycle, which becomes stable for large $Q$ before its transition to HSS via infinite period bifurcation. It is still not clear why phase-repulsive coupling permits the formation of APLC/IPLC switching. Intuitively it may be due to the increase in repressilator "stiffness" for large values of $n$. Similar frequency trigger covering a wide range of parameters was observed in the system of two FitzHugh-Nagumo oscillators coupled via the recovery variable if their stiffness was large [37].

All the results discussed above have been obtained for sets with different $\beta_{i}$. The oscillators are identical but move along the partial limit cycles nonuniformly because the kinetics of the specific variable in one element of the ring is faster than in the other elements. Variation in $\beta_{i}$ is biologically motivated given that there are parameters' differences between the three repressilator genes, their promoters, and degradation rates of the corresponding transcription factors. This internal variety in $\beta_{i}$ may enhance the coupling-induced diversity of the attractors. To check this possibility we simulated the dynamic behavior in the 3-dim parameter cube $(n, \alpha, \kappa)$ using several sets of $\beta_{i}$ varying in the degree of equalization (e.g., 0.25 , $0.15,0.15 ; 0.3,0.25,0.15)$ (data not shown). The only general effect of smoothing the $\beta_{i}$ is the necessity to tune reasonably the other parameters, but the qualitative picture of the phase diagrams is not changed.

We suggest that our results obtained from the coupling of two oscillators may have relevance to small populations of

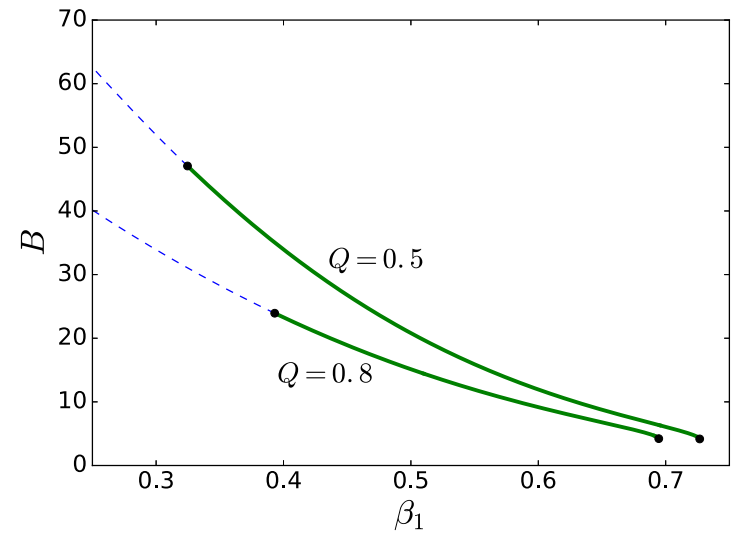

FIG. 23. $\beta_{1}$ continuations of APLC branch, stable (green) and unstable (blue dashed). $n=2.8, \kappa=17, \alpha=204, \beta_{2}=\beta_{3}=0.1$.
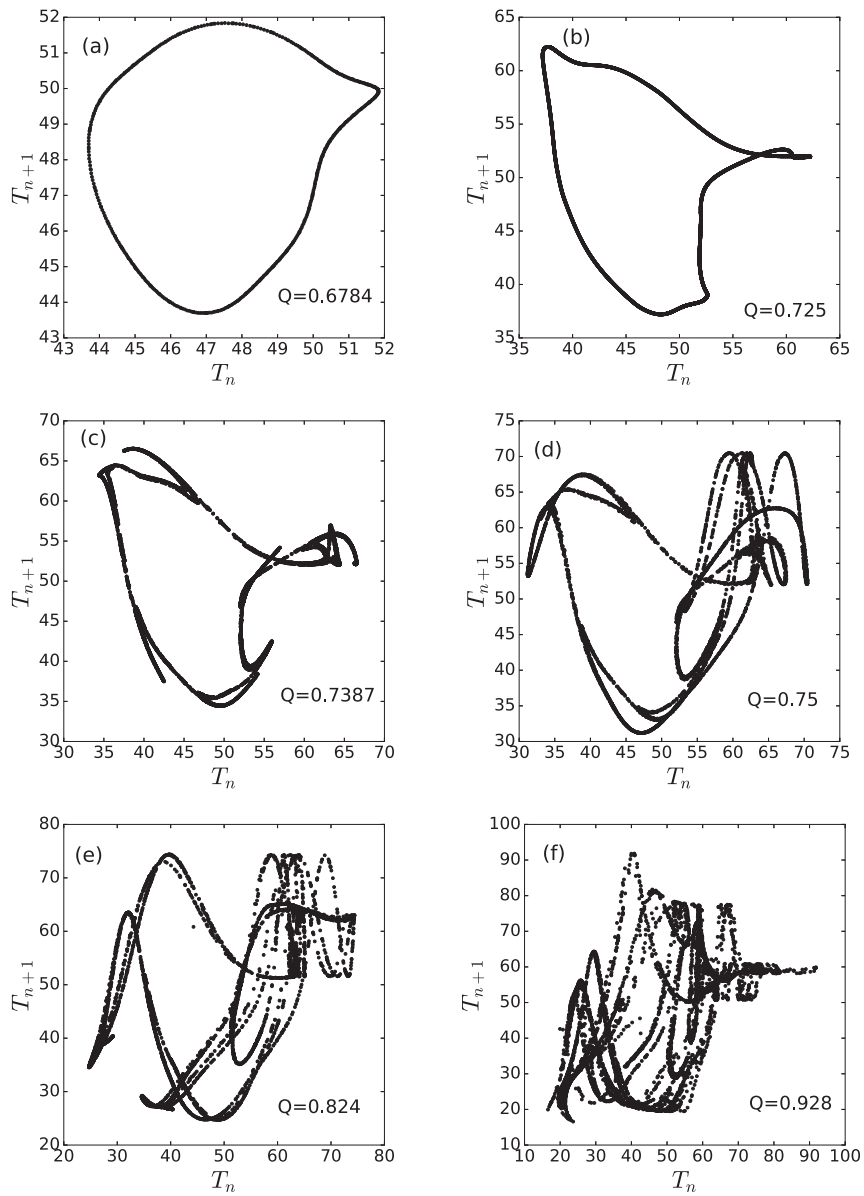

FIG. 24. Evolution of sequential period maps: (a) $Q=0.6784$ (quasiperiodic regime), (b) 0.725 (simple chaos), (c) 0.7387, (d) 0.75, (e) 0.824 , (f) 0.928. $n=3.15, \alpha=225, \kappa=10$.

cells. This idea is based on the phenomenon of clustering which is typical of global repulsive coupling. It has been shown [24] that for a particular set of parameters the population of QS-coupled identical or nearly identical repressilators splits into exactly three clusters. The oscillations inside clusters are in-phase synchronized and what is different is the number of oscillators in the clusters. The reduction in dimension to three clusters allows us to suggest that the variety of dynamics reported here may also apply (with possible modifications) to a more or less homogeneous population.

No doubt, the model in Eq. (1) is a strongly reduced dynamic toy which has limited experimental support for

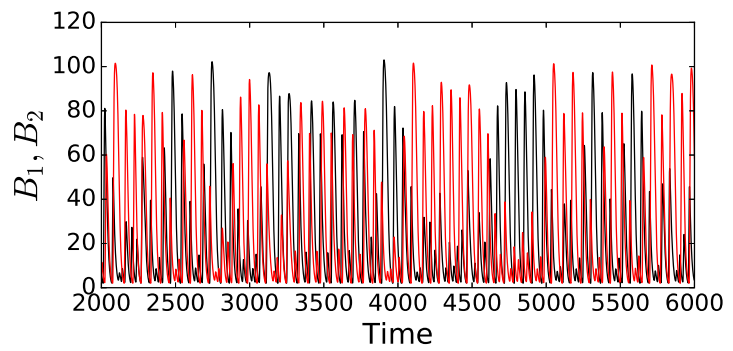

FIG. 25. Complex oscillations time series for $Q=0.928, n=$ $3.15, \alpha=225, \kappa=10$. Pieces of As1:2LC and 3:3LC are evident. 


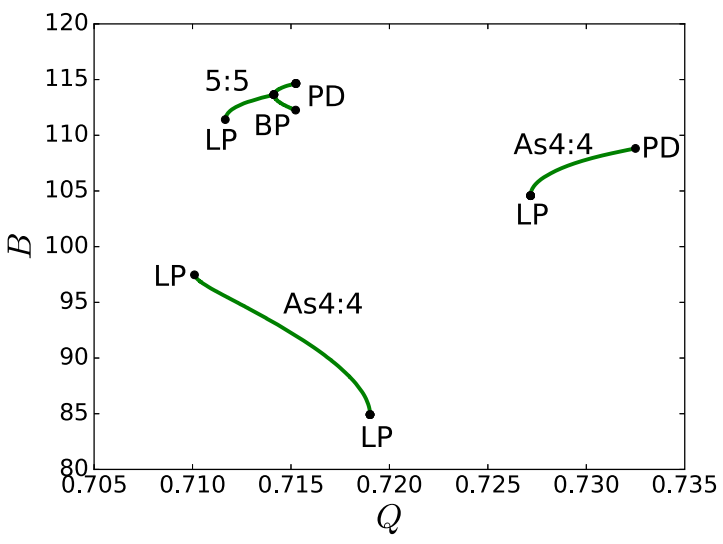

FIG. 26. Bifurcation diagram showing coexistence of As4:4LC and the stable end of a 5:5LC, in addition to the stable end of a different As4:4 branch.

formulation of exact forms of functions for the basic repressilators' ring and especially for the subsystem describing coupling. However, the general design formalized in Eq. (1) is realistic and realizable by the methods of synthetic genetics. Therefore, we propose that a pair of coupled repressilators may be viewed as a prototype of a flexible generator with remarkable dynamic behavior which may be useful beyond the construction of synthetic genetic networks.

\section{ACKNOWLEDGMENTS}

We thank the reviewers for valuable suggestions. This work was partially supported by Russian Foundation for Basic Research, Grants No. 15-02-03236 and No. 14-01-00196 to E.V.

\section{APPENDIX A: ELECTRONIC MODEL}

Here we give a brief description of how the electronic circuit models the two QS-coupled repressilators. Detailed explanation is given elsewhere [21,22]. Figure 20 shows the circuit for a single gene with expressed protein output $V_{i}$ and inhibition by $V_{i-1}$ at the input. Increasing the inhibitor $V_{i-1}$ raises the transistor's base voltage thereby shutting it off (inhibiting protein expression). In this way the circuit mimics the Hill function inhibition $1 /\left(1+x^{n}\right)$ as demonstrated in Fig. 2(a). Our previous circuit analysis [22] shows that it
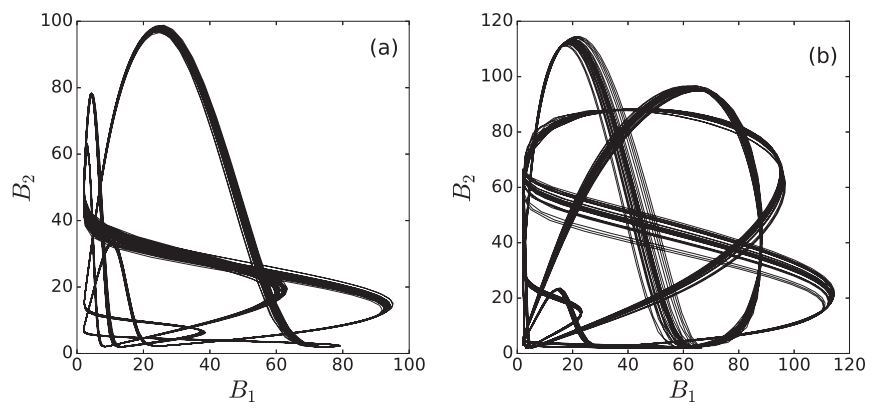

FIG. 27. Phase portraits of coexisting limit cycles in periodic windows at $Q=0.714$ in the presence of additional noise. (a) Asymmetrical 4:4LC. (b) Symmetric 5:5LC. $n=3.15, \alpha=$ $225, \kappa=10$. is convenient to vary model parameters while keeping the product $n \alpha$ constant. Therefore, we select a desired value of $n$ by changing $\alpha$, which is set by the reference voltage $V_{\text {cth }}$ in Fig. 20. Adjusting $V_{\text {cth }}$ matches the position and slope of inhibition in the circuit to the $50 \%$ inhibition in the Hill repression $1 /\left(1+x^{n}\right)$ at $x=1$. A second circuit value in Fig. 20, resistor $R_{\text {hill }}$, controls the repression for $x>1$. The ability to vary the two values $V_{\text {cth }}$ and $R_{\text {hill }}$ gives more flexibility in adjusting the shape of the repression curve for the circuit than does adjusting the single parameter $n$ in the Hill function. Table I shows some model parameter pairs $(n, \alpha)$ and the circuit values $V_{t h}$ and $R_{\text {hill }}$ used here to produce them. As with other details, the connection between $V_{t h}$ and $V_{\text {cth }}$ is explained elsewhere [22].

The circuit for a repressilator with QS feedback is shown in Fig. 21. The repressilator consists of the closed gene-gene loop with op-amp buffers between the genes. Each "gene" triangle contains the circuit in Fig. 20. The QS circuitry takes input from current source $I(B)$ controlled by the repressilator's Bprotein voltage and feeds back to the repressilator's C-protein via source $I(S)$. Source $I(B)$ accounts for the term $k_{s 1} B$ in Eq. (1d), and source $I(S)$ accounts for the activation term $\kappa \min (0.8 S, 1)$ in Eq. (3). Figure 2(b) shows how well $I(S)$ matches the activation term.

Figure 21 also shows how voltages $S_{1}$ and $S_{2}$ representing the autoinducer (AI) concentrations from two coupled repressilators combine to produce the external AI concentration
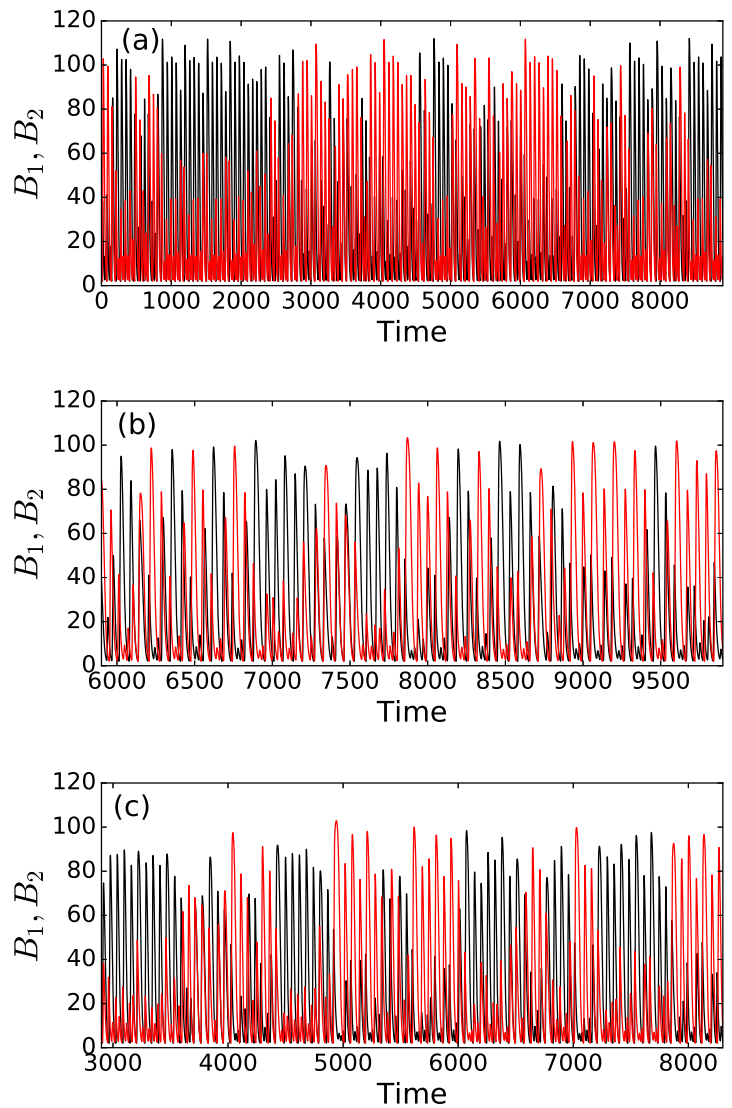

FIG. 28. Time series of chaos composed of alternating polarities of asymmetric LC. (a) $Q=0.82$ has As2:3LC pieces, (b) $Q=0.925$, and (c) $Q=0.95$ have a variety of AsLC pieces. 


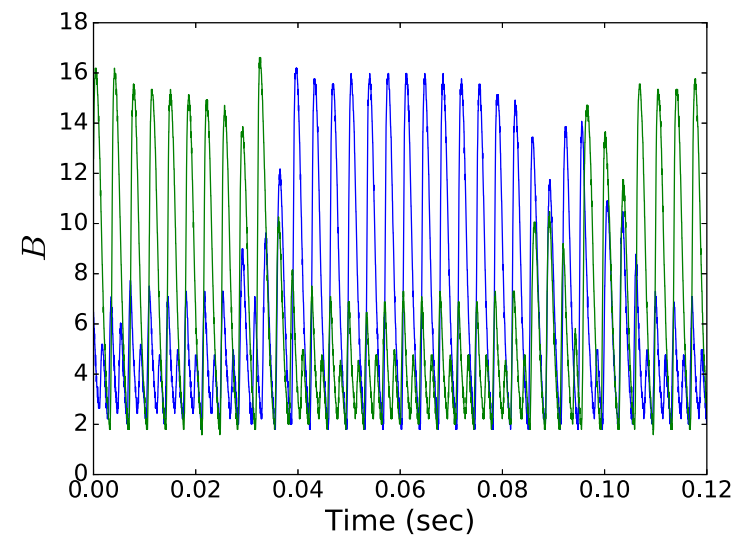

FIG. 29. Switching "polarities" of unstable As1:2LC measured in circuit. $n=3.2, \alpha=148, \kappa=22, Q=0.9$.

voltage $S_{\text {ext }}$. The dilution factor $Q$ [Eq. (2)] is controlled by resistor $R_{Q}$ and the diffusion constant [ $\eta$ in Eq. (1d)] is controlled by $R_{d}$. The current sources $I(B)$ and $I(S)$, the setting of initial conditions, and the control of all other model parameters by circuit values are described elsewhere [21,22].

\section{APPENDIX B: ADDITIONAL RESULTS}

\section{Low repression: $n=\mathbf{2 . 8}$}

Here we investigate the sensitivity of the multistability shown in Fig. 3 to changes in parameter value. Figure 22 shows that when $\alpha$ is reduced to 180 or increased to 250 the structure of the dynamics remains the same as Fig. 3 for $\alpha=204$. The multistability is not restricted to a narrow range of $\alpha$.

For $n=2.8$ with $\beta_{1}=0.5, \beta_{2}=\beta_{3}=0.1$ the dominant oscillatory behavior over a broad $Q$ range is stable APLC. Figure 23 shows $\beta_{1}$ continuations of the APLC. Decreasing $\beta_{1}$ in order to make the $\beta$ values closer causes the APLC to become unstable. However, calculations show no new dynamics are found not already seen for $\beta_{1}=0.5$ at higher $n$-values.

\section{High repression: $n=3.15$ \\ a. Evolution of sequential period maps}

The evolution of "sequential period maps" $T(n+1)$ versus $T(n)$ where $T(n)$ are the return times (subperiods) for a given Poincaré section are shown in Fig. 24. Only easily identified qualitative changes in the structures of maps are presented here. The changes in these maps may be compared with their locations on the map of regimes in Fig. 18. Figures 24(a)-24(c) correspond to the region just beyond the TR bifurcation where quasiperiodic beating develops into "simple chaos" (no highly

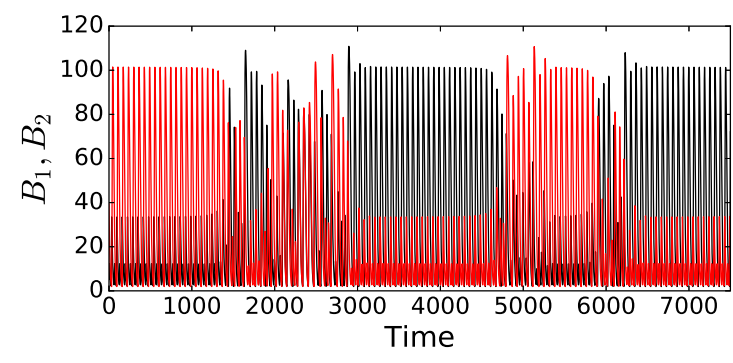

FIG. 30. $Q=0.8368$ Intermittency near LP of As1:2LC.

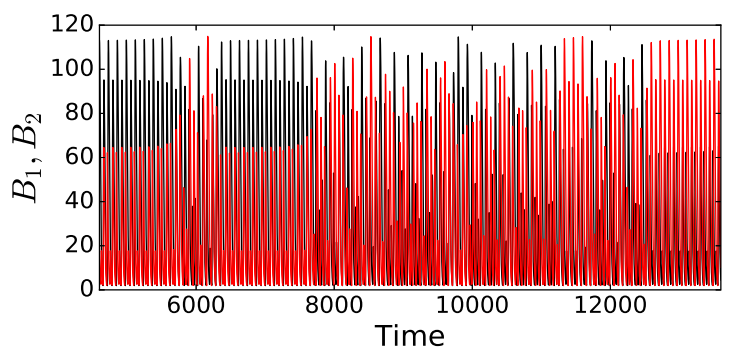

FIG. 31. $Q=0.7528$. Intermittency near LP of As2:3LC.

asymmetric pieces). Further increase of $Q$ introduces highly asymmetric n:m limit cycles (As2:3LC followed by As1:2LC) and symmetric n:n limit cycles. The unstable portions of these additional limit cycles provide more types of dynamics to the $\mathrm{CO}$, resulting in the increasingly complex sequential period maps in Figs. 24(d)-24(f). For example, for $Q=0.928$ the time series shown in Fig. 25 contains pieces of As1:2LC and 3:3LC.

\section{b. Periodic windows in simple chaos}

After the TR bifurcation in Fig. 18 there are many expected periodic windows each of which contains high period $n: n$ antiphase limit cycle. Apart from them, on the route to chaos presented above (Fig. 24) there is an interesting window with stable asymmetrical limit cycle marked As4:4LC in the $Q$ continuation shown in Fig. 26 (zoom of Fig. 18) along with a coexisting stable end of a 5:5LC and a stable end of a different As4:4LC. Although the parameter's intervals with stable attractor are very narrow (see Fig. 18), the As4:4LC regime is unusual because it is pure asymmetrical (confined by LP), and it coexists with symmetrical 5:5LC ( $Q=0.7116-0.715)$. (The other As4:4LC in Fig. 26 is also pure asymmetrical, undergoing PD cascade to chaos followed by period-halving back to stable As4:4LC at $Q=1.267$.) Phase portraits of the coexisting 5:5LC and As4:4LC with noise are shown in Fig. 27. Simulations in the presence of noise show that the As4:4LC is significantly less sensitive to noise than the 5:5LC, which demonstrates noise-induced switching to the As 4:4LC under very small noise. We did not observe the reverse switching, suggesting that the 5:5LC has a small basin of attraction compared to the As4:4LC.

\section{c. Alternating polarity chaos}

Figure 28 shows examples of typical time series which illustrate the alternating "polarity" of oscillators in chaotic regime. This switching behavior occurs when the system lands near an unstable AsLC and takes a number of cycles to fall off it, then returns with the roles of the two oscillators switched.

An example of alternating As 1:2LC polarities measured in a circuit is shown in Figure 29.

\section{d. Intermittency}

Intermittency may occur in a regime just outside of a stable limit cycle. The behavior is characterized by durations of the LC interspersed with COs. Figure 30 shows intermittency for $Q$ just below the LP of the As1:2LC and Fig. 31 near the LP of As2:3LC. 
[1] J. J. Tyson and B. Novák, Annu. Rev. Phys. Chem. 61, 219 (2010).

[2] M. B. Elowitz and S. Leibler, Nature (London) 403, 335 (2000).

[3] A. Loinger and O. Biham, Phys. Rev. E 76, 051917 (2007).

[4] O. Buse, R. Pérez, and A. Kuznetsov, Phys. Rev. E 81, 066206 (2010).

[5] N. Strelkowa and M. Barahona, J. R. Soc. Interf. 7, 1071 (2010).

[6] C. M. Waters and B. L. Bassler, Annu. Rev. Cell Dev. Biol. 21, 319 (2005).

[7] F. K. Balagadde, H. Song, J. Ozaki, C. H. Collins, M. Barnet, F. H. Arnold, S. R. Quake, and L. You, Mol. Syst. Biol. 4, 187 (2008).

[8] L. You, R. S. Cox, R. Weiss, and F. H. Arnold, Nature (London) 428, 868 (2004).

[9] S. Hennig, G. Rödel, and K. Ostermann, J. Biol. Eng. 9, 13 (2015).

[10] D. McMillen, N. Kopell, J. Hasty, and J. J. Collins, Proc. Natl. Acad. Sci. USA 99, 679 (2002).

[11] J. Garcia-Ojalvo, M. B. Elowitz, and S. H. Strogatz, Proc. Nat. Acad. Sci. USA 101, 10955 (2004).

[12] A. Kuznetsov, M. Kærn, and N. Kopell, SIAM J Appl. Math. 65, 392 (2004).

[13] T. Danino, O. Mondragon-Palomino, L. Tsimring, and J. Hasty, Nature (London) 463, 326 (2010).

[14] Y. Chen, J. K. Kim, A. J. Hirning, K. Josić, and M. R. Bennett, Science 349, 986 (2015).

[15] T. Zhou, J. Zhang, Z. Yuan, and L. Chen, Chaos 18, 037126 (2008).

[16] E. Conrad, A. E. Mayo, A. J. Ninfa, and D. B. Forger, J. R. Soc. Interf. 5, S9 (2008).

[17] A. Wagemakers, J. M. Buldú, J. García-Ojalvo, and M. A. F. Sanjuán, Chaos 16, 013127 (2006).
[18] I. Potapov, E. Volkov, and A. Kuznetsov, Phys. Rev. E 83, 031901 (2011)

[19] E. Ullner, A. Zaikin, E. I. Volkov, and J. Garcia-Ojalvo, Phys. Rev. Lett. 99, 148103 (2007).

[20] E. Ullner, A. Koseska, J. Kurths, E. Volkov, H. Kantz, and J. Garcia-Ojalvo, Phys. Rev. E 78, 031904 (2008).

[21] E. H. Hellen, E. Volkov, J. Kurths, and S. K. Dana, PLoS ONE 6, e23286 (2011)

[22] E. H. Hellen and S. K. Dana, arXiv:1607.06510 [physics.bio$\mathrm{ph}]$.

[23] E. H. Hellen, S. K. Dana, B. Zhurov, and E. Volkov, PLoS ONE 8, e62997 (2013).

[24] A. Koseska, E. Ullner, E. Volkov, J. Kurths, and J. GarcíaOjalvo, J. Theor. Biol. 263, 189 (2010).

[25] M. Toiya, V. K. Vanag, and I. R. Epstein, Angew. Chem., Int. Ed. Engl. 47, 7753 (2008).

[26] B. Ermentrout, Simulating, Analyzing, and Animating Dynamical Systems: A Guide to XPPAUT for Researchers and Students (SIAM, Philadelphia, 2002).

[27] A. Prindle, J. Selimkhanov, H. Li, I. Razinkov, L. S. Tsimring, and J. Hasty, Nature (London) 508, 387 (2014).

[28] O. Rössler, Z. Naturforsch. 31 a, 1168 (1976).

[29] H. Fujisaka and T. Yamada, Z. Phys. B 37, 265 (1980).

[30] I. Schreiber and M. Marek, Physica D 5, 258 (1982).

[31] C. G. Hocker and I. R. Epstein, J. Chem. Phys. 90, 3071 (1989).

[32] I. Waller and R. Kapral, Phys. Lett. A 105, 163 (1984).

[33] G. N. Borisyuk, R. M. Borisyuk, A. I. Khibnik, and D. Roose, Bull. Math. Biol. 57, 809 (1995).

[34] S. K. Han and D. E. Postnov, Chaos 13, 1105 (2003).

[35] P.-C. Romond, M. Rustici, D. Gonze, and A. Goldbeter, Ann. NY Acad. Sci. 879, 180 (1999).

[36] O. Sporns, S. Roth, and F. F. Seelig, Physica D 26, 215 (1987).

[37] E. Volkov and M. Stolyarov, Phys. Lett. A 159, 61 (1991). 